Pacific

Journal of

Mathematics

CATEGORIES OF UNITARY REPRESENTATIONS OF BANACH-LIE SUPERGROUPS AND RESTRICTION FUNCTORS

StéPhane Merigon, Karl-Hermann NeEb and Hadi Salmasian 


\title{
CATEGORIES OF UNITARY REPRESENTATIONS OF BANACH-LIE SUPERGROUPS AND RESTRICTION FUNCTORS
}

\author{
Stéphane Merigon, Karl-Hermann NeEb and Hadi Salmasian
}

\begin{abstract}
We prove two results which show that the categories of smooth and analytic unitary representations of a Banach-Lie supergroup are well-behaved. The first result states that the restriction functor corresponding to any homomorphism of Banach-Lie supergroups is well-defined. The second result shows that the category of analytic representations is isomorphic to a subcategory of the category of smooth representations. These facts are needed as a crucial first step to a rigorous treatment of the analytic theory of unitary representations of Banach-Lie supergroups. They extend the known results for finite-dimensional Lie supergroups. In the infinite-dimensional case the proofs require several new ideas. As an application, we give an analytic realization of the oscillator representation of the restricted orthosymplectic Banach-Lie supergroup.
\end{abstract}

\section{Introduction}

In the last two decades, unitary representations of finite and infinite-dimensional Lie supergroups and Lie superalgebras have received growing interest from both mathematicians and physicists. These unitary representations appear in the classification of free relativistic superparticles in SUSY quantum mechanics (for example, see [Ferrara et al. 1981] and [Salam and Strathdee 1974]) which relies on the little supergroup method, an idea originating from the classical works of Mackey and Wigner. Unitary representations of the $N=1$ super Virasoro algebras were classified by Friedan, Qiu and Shenker [Friedan et al. 1985], Goddard, Kent and Olive [Goddard et al. 1986], and Sauvageot [1989]. For the $N=2$ super Virasoro algebras, the results are due to Boucher, Friedan, Kent [Boucher et al. 1986] and Iohara [2010]. Kac and Todorov [1985] classified unitary representations of superconformal current algebras. Using an analogue of the Sugawara construction, Jarvis and Zhang [1988] constructed unitary representations of untwisted affine Lie

Salmasian was supported by a Discovery Grant from the Natural Sciences and Engineering Research Council of Canada and an Alexander von Humboldt Fellowship for Experienced Researchers.

MSC2010: 17B65, 22E66.

Keywords: Banach-Lie supergroups, unitary representations, smooth vectors, analytic vectors. 
superalgebras. Unitary highest weight modules of affine Lie superalgebras were also studied by Jakobsen and Kac [1989].

Much of the research done on unitary representations is algebraic, that is, studies them as unitarizable modules over Lie superalgebras. A mathematically rigorous investigation of analytic aspects of unitary representations is more recent [Alldridge et al. 2011; Carmeli et al. 2006; Salmasian 2010].

Carmeli et al. [2006] propose an approach to harmonic analysis on Lie supergroups. One key idea in their work is to use the equivalence between the category of Lie supergroups and the category of Harish-Chandra pairs ${ }^{1}(G, \mathfrak{g})$ [Deligne and Morgan 1999, Section 3.8; Kostant 1977, Section 3.2]. The advantage of using this equivalence is that for Harish-Chandra pairs the definition of a unitary representation is more concrete. Roughly speaking, a Harish-Chandra pair is an ordered pair $(G, \mathfrak{g})$, where $G$ is a Lie group, $\mathfrak{g}=\mathfrak{g}_{\overline{0}} \oplus \mathfrak{g}_{\overline{1}}$ is a Lie superalgebra, $\mathfrak{g}_{\overline{0}}=\operatorname{Lie}(G)$, and there is an action of $G$ on $\mathfrak{g}$ which is compatible with the adjoint action of $\mathfrak{g}$. (For a precise definition, see Definition 2.1.) A unitary representation of $(G, \mathfrak{g})$ is a triple $\left(\pi, \mathscr{H}, \rho^{\pi}\right)$, where $(\pi, \mathscr{H})$ is a unitary representation of the Lie group $G$ (in the sense of [Varadarajan 1999, Section 1.2]) and $\rho^{\pi}$ is a representation of the Lie superalgebra $\mathfrak{g}$, realized on a dense subspace of $\mathscr{H}$ (consisting of smooth vectors) which is compatible with $\pi$ on $\mathfrak{g}_{\overline{0}}$ (see Definition 4.1).

An important observation in [Carmeli et al. 2006] is that if $x \in \mathfrak{g}_{\overline{1}}$ then

$$
\rho^{\pi}(x)^{2}=\frac{1}{2} \rho^{\pi}([x, x])=\frac{1}{2} \mathrm{~d} \pi([x, x]),
$$

which suggests that $\rho^{\pi}(x)$ should be an unbounded operator on $\mathscr{H}$, that is, it can only be densely defined. Therefore one needs to fix a common domain for the operators $\rho^{\pi}(x)$. For instance, one can choose the common domain to be $\mathscr{H}^{\infty}$ or $\mathscr{H}^{\omega}$, the subspaces of smooth or analytic vectors of the unitary representation $(\pi, \mathscr{H})$, which lead to categories $\operatorname{Rep}^{\infty}(G, \mathfrak{g})$ and $\operatorname{Rep}^{\omega}(G, \mathfrak{g})$ of smooth and analytic representations of $(G, \mathfrak{g})$. With any choice of such a common domain, we are led to the following two questions.

(i) What is the relation between the categories of unitary representations of $(G, \mathfrak{g})$ when the common domain for the realization of $\rho^{\pi}$ varies? For instance, what is the relation between $\operatorname{Rep}^{\infty}(G, \mathfrak{g})$ and $\operatorname{Rep}^{\omega}(G, \mathfrak{g})$ ?

Here the issue is that if $\left(\pi, \rho^{\pi}, \mathscr{H}\right)$ is an object of $\operatorname{Rep}^{\omega}(G, \mathfrak{g})$, then the action of $\mathfrak{g}_{1}$ is defined on $\mathscr{H}^{\omega}$. In general $\mathscr{H}^{\omega} \subsetneq \mathscr{H}^{\infty}$ and therefore a priori it is not obvious why $\left(\pi, \rho^{\pi}, \mathscr{H}\right)$ is also an object of $\operatorname{Rep}^{\infty}(G, \mathfrak{g})$.

\footnotetext{
${ }^{1}$ Because of the equivalence between the categories of Lie supergroups and Harish-Chandra pairs, it will be harmless (and simpler for our presentation) to refer to a Harish-Chandra pair as a Lie supergroup. When the Harish-Chandra pair is modeled on a Banach space, we will call it a BanachLie supergroup (see Definition 2.1).
} 
(ii) Given a homomorphism of Banach-Lie supergroups $(\phi, \varphi):(H, \mathfrak{h}) \rightarrow(G, \mathfrak{g})$, are there well-defined restriction functors

$$
\operatorname{Res}^{\infty}: \operatorname{Rep}^{\infty}(G, \mathfrak{g}) \mapsto \operatorname{Rep}^{\infty}(H, \mathfrak{h})
$$

and

$$
\operatorname{Res}^{\omega}: \operatorname{Rep}^{\omega}(G, \mathfrak{g}) \mapsto \operatorname{Rep}^{\omega}(H, \mathfrak{h}) ?
$$

Here the issue is the following. Let $\left(\pi, \rho^{\pi}, \mathscr{H}\right)$ be a unitary representation of $(G, \mathfrak{g})$. Denote the subspace of smooth vectors of $(\pi, \mathscr{H})$ (respectively, $(\pi \circ \phi, \mathscr{H})$ ) by $\mathscr{H}_{G}^{\infty}$ (respectively, $\left.\mathscr{H}_{H}^{\infty}\right)$. A priori the operators $\rho^{\pi} \circ \varphi(x)$, where $x \in \mathfrak{h}_{\overline{1}}$, are only defined on $\mathscr{H}_{G}^{\infty}$. In general $\mathscr{H}_{G}^{\infty} \subsetneq \mathscr{H}_{H}^{\infty}$, and restricting the actions naively does not lead to an object of the category $\operatorname{Rep}^{\infty}(H, \mathfrak{h})$.

The answers to the above questions are crucial to obtaining well-behaved categories of unitary representations for Harish-Chandra pairs. For (finite-dimensional) Lie supergroups they are addressed in [Carmeli et al. 2006].

The main goal of this article is to answer the above questions for Harish-Chandra pairs associated to Banach-Lie supergroups. Molotkov's work (which is extended in Sachse's Ph.D. thesis) develops a functorial theory of Banach-Lie supermanifolds which specializes to the (finite-dimensional) Berezin-Leites-Kostant theory and the (possibly infinite-dimensional) DeWitt-Tuynman theory [Alldridge and Laubinger 2012]. In the functorial approach one can associate Harish-Chandra pairs to Banach-Lie supergroups as well. We believe that the success of the HarishChandra pair approach in the finite-dimensional case justifies their use in studying harmonic analysis on Banach-Lie supergroups.

The direction we choose for the formulation and proofs of our results is similar in spirit to [Carmeli et al. 2006]. However, some of the arguments used in that source, in particular the proofs of its Propositions 1 and 2, depend crucially on finiteness of dimension. In this article we present new arguments which generalize to the Banach-Lie case. One of our tools is the theory of analytic maps between Banach spaces. Of the multitude of existing variations of this theory, the most relevant to this article is the work in [Bochnak and Siciak 1971a; 1971b; Siciak 1972], which generalizes the results in [Hille and Phillips 1974].

We also present two applications of our techniques. The first application is that when $g$ is in the connected component of identity of $G$, the conjugacy invariance relation

$$
\pi(g) \rho^{\pi}(x) \pi(g)^{-1}=\rho^{\pi}(\operatorname{Ad}(g) x) \quad \text { for every } x \in \mathfrak{g}
$$

follows from the remaining assumptions in the definition of a unitary representation of $(G, \mathfrak{g})$. (See Proposition 4.8 below.) Such a conjugacy invariance relation is one 
of the assumptions of [Carmeli et al. 2006, Definition 2]. As a consequence, we obtain a reformulation of the definition of a unitary representation (see Definition 4.1) which makes it more practical than the original form given in [Carmeli et al. 2006] because in explicit examples checking that the infinitesimal action satisfies the bracket relation is easier than checking the conjugacy invariance relation (1).

The second application is an analytic realization of the oscillator representation of the restricted orthosymplectic Banach-Lie supergroup. Again the main issue is to show that the action of the odd part is defined on the subspaces of smooth and analytic vectors and leaves them invariant. We use a general statement, that is, Theorem 4.10, which we expect to be useful in a variety of situations, for instance when one is interested in integrating a representation of a Banach-Lie superalgebra.

This article is organized as follows. In Section 2 we introduce our notation and basic definitions and prove some general lemmas which will be used in the later sections. In Section 3 we state some general facts about smooth and analytic vectors of unitary representations of Banach-Lie groups. Section 4 is devoted to the proof of our main results, Theorems 4.10 and 4.12. In Section 5 we give a realization of the oscillator representation of the restricted orthosymplectic Banach-Lie supergroup. In Appendix A we give an example of a smooth unitary representation of a Banach-Lie group which has no nonzero analytic vectors. In Appendix B we give an example of an analytic unitary representation of a Banach-Lie group which has no nonzero bounded vectors. Appendix $\mathrm{C}$ contains the background material on analytic maps between Banach spaces.

This article contains general results about arbitrary Banach-Lie supergroups. These results are needed for the study of unitary representations of concrete examples. In our forthcoming works we will study unitary representations of BanachLie supergroups corresponding to affine Lie superalgebras, and the supergroup version of the Kirillov-Ol'shanskii classification of unitary representations of the infinite-dimensional unitary group [Kirillov 1973; Ol'shanskii 1991]. We also plan to investigate unitary representations of super versions of loop groups, resp., suitable double extensions. On the algebraic side, a classification of the unitary highest weight modules are known from the work of Kac and Todorov [1985] and Jarvis and Zhang [1988]. To develop a suitable analytic context, we plan to use Lie supergroups $(G, \mathfrak{g})$, where $G$ is a Banach-Lie group of $H^{1}$-loops with values in a compact Lie algebra, so that we can build on the results developed in the present paper.

\section{Notation and preliminaries}

If $\mathscr{B}$ is a real Banach space with norm $\|\cdot\|_{\mathbb{R}}$ then we define its complexification as the complex Banach space with underlying space $\mathscr{B}^{\mathbb{C}}=\mathscr{B}_{B} \otimes_{\mathbb{R}} \mathbb{C}$ and with norm 
$\|\cdot\|_{\mathbb{C}}$, where for every $v \in \mathscr{B}^{\mathbb{C}}$ we set

$$
\|v\|_{\mathbb{C}}=\inf \left\{\left|\zeta_{1}\right| \cdot\left\|v_{1}\right\|_{\mathbb{R}}+\cdots+\left|\zeta_{k}\right| \cdot\left\|v_{k}\right\|_{\mathbb{R}}: v=v_{1} \otimes_{\mathbb{R}} \zeta_{1}+\cdots+v_{k} \otimes_{\mathbb{R}} \zeta_{k}\right\}
$$

Note that if $v=v_{1} \otimes_{\mathbb{R}} 1+v_{2} \otimes_{\mathbb{R}} i$ then

$$
\max \left\{\left\|v_{1}\right\|_{\mathbb{R}},\left\|v_{2}\right\|_{\mathbb{R}}\right\} \leq\|v\|_{\mathbb{C}} \leq\left\|v_{1}\right\|_{\mathbb{R}}+\left\|v_{2}\right\|_{\mathbb{R}} .
$$

All Banach and Hilbert spaces will be separable. Let $\mathscr{H}$ be a real or complex Hilbert space. If $T: \mathscr{H} \rightarrow \mathscr{H}$ is a bounded linear operator on $\mathscr{H}$, then $\|T\|_{\text {Op }}$ denotes the operator norm of $T$, and if $T$ is a Hilbert-Schmidt operator, then $\|T\|_{\mathrm{HS}}$ denotes its Hilbert-Schmidt norm.

Now assume $\mathscr{H}$ is a complex Hilbert space. The group of unitary linear transformations on $\mathscr{H}$ is denoted by $\mathrm{U}(\mathscr{H})$. The domain of an unbounded linear operator $T$ on $\mathscr{H}$ is denoted by $\mathscr{D}(T)$, and if $\mathscr{B} \subseteq \mathscr{D}(T)$ is a subspace, then $\left.T\right|_{\mathscr{B}}$ denotes the restriction of $T$ to $\mathscr{B}$. If $S$ and $T$ are two unbounded operators on $\mathscr{H}$ then their sum $S+T$ is an operator with domain $\mathscr{D}(S+T)=\mathscr{D}(S) \cap \mathscr{D}(T)$, and their product $S T$ is an operator with domain

$$
\mathscr{D}(S T)=\{v \in \mathscr{D}(T): T v \in \mathscr{D}(S)\} .
$$

For two unbounded operators $S$ and $T$, we write $S \prec T$ if $\mathscr{D}(S) \subseteq \mathscr{D}(T)$ and $\left.T\right|_{\mathscr{D}(S)}=S$.

The adjoint of a linear operator $T$ is denoted by $T^{*}$. If $T$ is closable, then its closure is denoted by $\bar{T}$. For every integer $n>1$ we set

$$
\mathscr{D}\left(T^{n}\right)=\left\{v \in \mathscr{D}(T): T v \in \mathscr{D}\left(T^{n-1}\right)\right\} .
$$

We also set $\mathscr{D}^{\infty}(T)=\bigcap_{n=1}^{\infty} D\left(T^{n}\right)$. If $v \in \mathscr{D}^{\infty}(T)$ satisfies

$$
\sum_{n=0}^{\infty} \frac{t^{n}}{n !}\left\|T^{n} v\right\|<\infty \quad \text { for some } t>0
$$

then $v$ is called an analytic vector of $T$. The space of analytic vectors of $T$ is denoted by $\mathscr{D}^{\omega}(T)$.

By a $\mathbb{Z}_{2}$-graded Hilbert space $\mathscr{H}=\mathscr{H}_{\overline{0}} \oplus \mathscr{H}_{\overline{1}}$ we simply mean an orthogonal direct sum of two complex Hilbert spaces $\mathscr{H}_{\overline{0}}$ and $\mathscr{H}_{\overline{1}}$. (For another equivalent definition, see [Carmeli et al. 2006, Section 2.1].)

All Banach-Lie groups and the homomorphisms between them will be real analytic. Let $G$ be a Banach-Lie group and $\mathfrak{g}=\operatorname{Lie}(G)$. The identity component of $G$ is denoted by $G^{\circ}$. We assume, without loss of generality, that the norm inducing the topology of the Banach-Lie algebra $\mathfrak{g}$ satisfies $\|[x, y]\| \leq\|x\| \cdot\|y\|$ for every $x, y \in \mathfrak{g}$. 
By a Banach-Lie superalgebra we mean a Lie superalgebra $\mathfrak{g}=\mathfrak{g}_{\overline{0}} \oplus \mathfrak{g}_{\overline{1}}$ over $\mathbb{R}$ or $\mathbb{C}$ with the following two properties.

(i) $\mathfrak{g}$ is a Banach space with a norm $\|\cdot\|$ satisfying

$$
\|[x, y]\| \leq\|x\| \cdot\|y\| \quad \text { for every } x, y \in \mathfrak{g} .
$$

(ii) $\mathfrak{g}_{\overline{0}}$ and $\mathfrak{g}_{\overline{1}}$ are closed subspaces of $\mathfrak{g}$.

Remark. Consider the norm $\|\cdot\|^{\prime}$ on $\mathfrak{g}$ which is defined as follows. For every $x \in \mathfrak{g}$, we write $x=x_{\overline{0}}+x_{\overline{1}}$, where $x_{\overline{0}} \in \mathfrak{g}_{\overline{0}}$ and $x_{\overline{1}} \in \mathfrak{g}_{\overline{1}}$, and set $\|x\|^{\prime}=\left\|x_{\overline{0}}\right\|+\left\|x_{\overline{1}}\right\|$. From the definition of a Banach-Lie superalgebra it follows that the norms $\|\cdot\|$ and $\|\cdot\|^{\prime}$ are equivalent.

The Banach-Lie group of continuous even automorphisms (that is, automorphisms which preserve parity) of a Banach-Lie superalgebra $\mathfrak{g}$ is denoted by $\operatorname{Aut}(\mathfrak{g})$.

Definition 2.1. A Banach-Lie supergroup is an ordered pair $(G, \mathfrak{g})$ with the following properties.

(i) $G$ is a Banach-Lie group.

(ii) $\mathfrak{g}$ is a Banach-Lie superalgebra over $\mathbb{R}$.

(iii) $\mathfrak{g}_{0}=\operatorname{Lie}(G)$.

(iv) There exists a homomorphism of Banach-Lie groups Ad: $G \rightarrow \operatorname{Aut}(\mathfrak{g})$ such that

$$
\mathrm{d}_{e} \operatorname{Ad}(x)=\operatorname{ad}_{x} \quad \text { for every } x \in \mathfrak{g}_{\overline{0}},
$$

where $\mathrm{d}_{e} \mathrm{Ad}$ denotes the differential of Ad at $e \in G$, and $\operatorname{ad}_{x}(y)=[x, y]$.

We refer to the homomorphism Ad : $G \rightarrow \operatorname{Aut}(\mathfrak{g})$ of Definition 2.1(iv) by the adjoint action of $G$ on $\mathfrak{g}$. Observe that the map

$$
G \times \mathfrak{g} \rightarrow \mathfrak{g}, \quad(g, x) \mapsto \operatorname{Ad}(g) x
$$

is analytic.

If $\mathfrak{g}$ is a real Lie superalgebra, then its complexification is denoted by $\mathfrak{g}^{\mathbb{C}}$. It is easily seen that if $\mathfrak{g}$ is a real Banach-Lie superalgebra, then $\mathfrak{g}^{\mathbb{C}}$ with the norm defined in the beginning of Section 2 is a complex Banach-Lie superalgebra. After a suitable scaling, we can assume that the norm $\|\cdot\|$ chosen on $\mathfrak{g}^{\mathbb{C}}$ satisfies

$$
\|[x, y]\| \leq\|x\| \cdot\|y\| \quad \text { for every } x, y \in \mathfrak{g}^{\mathbb{C}} .
$$

A homomorphism of Banach-Lie supergroups $(\phi, \varphi):(H, \mathfrak{h}) \rightarrow(G, \mathfrak{g})$ is a pair of maps where $\phi: H \rightarrow G$ is a homomorphism of Banach-Lie groups, $\varphi: \mathfrak{h} \rightarrow \mathfrak{g}$ is a continuous homomorphism of Lie superalgebras, and $\mathrm{d}_{e} \phi=\left.\varphi\right|_{\mathfrak{h}_{\overline{0}}}$. 
A unitary representation of a Banach-Lie group $G$ is an ordered pair $(\pi, \mathscr{H})$ such that $\pi: G \rightarrow \mathrm{U}(\mathscr{H})$ is a group homomorphism and for every $v \in \mathscr{H}$ the orbit map

$$
\pi^{v}: G \rightarrow \mathscr{H}, \quad \pi^{v}(g)=\pi(g) v
$$

is continuous. If $\phi: H \rightarrow G$ is a homomorphism of Banach-Lie groups, then the restriction of $(\pi, \mathscr{H})$ to $H$ is the unitary representation $(\pi \circ \phi, \mathscr{H})$ of $H$.

We conclude this section with a few lemmas about unbounded operators.

Lemma 2.2. Let $T$ be a self-adjoint operator on a complex Hilbert space $\mathcal{H}$ and $\mathscr{L} \subseteq \mathscr{D}(T)$ be a dense subspace of $\mathscr{H}$ satisfying at least one of the following properties.

(a) For every $t \in \mathbb{R}$, we have e $e^{i t T} \mathscr{L} \subseteq \mathscr{L}$.

(b) Every $v \in \mathscr{L}$ is an analytic vector for $T$.

Then $\left.T\right|_{\mathscr{L}}$ is essentially self-adjoint.

Proof. When (a) holds, the result follows from [Reed and Simon 1972, Theorem VIII.11]. When (b) holds, it is an immediate consequence of Nelson's Analytic Vector Theorem [Nelson 1959, Lemma 5.1].

The next lemma is obvious, but it will help us shorten several similar arguments.

Lemma 2.3. Let $P_{1}$ and $P_{2}$ be symmetric linear operators on a complex Hilbert space $\mathscr{H}$ and $v \in \mathscr{D}\left(P_{1}\right) \cap \mathscr{D}\left(P_{2}\right)$ such that $P_{1} v \in \mathscr{D}\left(P_{1}\right)$ and $P_{1}^{2} v=P_{2} v$. Then

$$
\left\|P_{1} v\right\| \leq\|v\|^{\frac{1}{2}} \cdot\left\|P_{2} v\right\|^{\frac{1}{2}} .
$$

Proof. $\left\|P_{1} v\right\|^{2}=\left|\left\langle P_{1} v, P_{1} v\right\rangle\right|=\left|\left\langle v, P_{1}^{2} v\right\rangle\right|=\left|\left\langle v, P_{2} v\right\rangle\right| \leq\|v\| \cdot\left\|P_{2} v\right\|$.

Lemma 2.4. Let $T$ be a self-adjoint operator on a complex Hilbert space $\mathscr{H}$. Let $\mathscr{L}$ be a dense subspace of $\mathscr{H}$ such that $\mathscr{L} \subseteq \mathscr{D}(T)$ and $T \mid \mathscr{L}$ is essentially self-adjoint, and $S$ be a symmetric operator such that $\mathscr{L} \subseteq \mathscr{D}(S), S \mathscr{L} \subseteq \mathscr{L}$, and $\left.S^{2}\right|_{\mathscr{L}}=\left.T\right|_{\mathscr{L}}$. Then $\left.S\right|_{\mathscr{L}}$ is essentially self-adjoint, $\overline{\left.S\right|_{\mathscr{L}}}=\bar{S}$, and $\bar{S}^{2}=T$.

Proof. The proof of the lemma is similar to that of [Carmeli et al. 2006, Lemma 1]. We spell it out for completeness.

Set $S_{1}=\left.S\right|_{\mathscr{L}}$. From $T=\overline{\left.T\right|_{\mathscr{L}}}=\overline{S_{1}^{2}}$ it follows that

$$
\langle T v, v\rangle \geq 0 \quad \text { for every } v \in \mathscr{D}(T) .
$$

By [Reed and Simon 1972, Theorem VIII.3], in order to prove that $S_{1}$ is essentially self-adjoint, it suffices to show that if

$$
S_{1}^{*} v=\lambda v
$$


for a nonzero $v \in \mathscr{D}\left(S_{1}^{*}\right)$ and a $\lambda \in \mathbb{C}$, then $\lambda \in \mathbb{R}$. If $v$ and $\lambda$ satisfy (6) then for every $w \in \mathscr{L}$ we have

$$
\left\langle S_{1}^{2} w, v\right\rangle=\left\langle S_{1} w, S_{1}^{*} v\right\rangle=\bar{\lambda}\left\langle S_{1} w, v\right\rangle=\bar{\lambda}\left\langle w, S_{1}^{*} v\right\rangle=\bar{\lambda}^{2}\langle w, v\rangle=\left\langle w, \lambda^{2} v\right\rangle .
$$

Therefore $v \in \mathscr{D}\left(\left(S_{1}^{2}\right)^{*}\right)$ and $\left(S_{1}^{2}\right)^{*} v=\lambda^{2} v$. But $T=(T \mid \mathscr{L})^{*}=\left(S_{1}^{2}\right)^{*}$ and in particular $T v=\left(S_{1}^{2}\right)^{*} v=\lambda^{2} v$. From (5) it follows immediately that $\lambda \in \mathbb{R}$. This completes the proof of essential self-adjointness of $S_{1}$.

Next observe that $\overline{S_{1}} \prec \bar{S} \prec S^{*} \prec S_{1}^{*}$. Since $S_{1}$ is essentially self-adjoint, we have $\overline{S_{1}}=S_{1}^{*}$ and therefore $\bar{S}=\overline{S_{1}}$. Since the operator $\overline{S_{1}}$ is self-adjoint, it follows from [Dunford and Schwartz 1988, Corollary XII.2.8] that ${\overline{S_{1}}}^{2}$ is also self-adjoint. Consequently,

$$
{\overline{S_{1}}}^{2}=\left({\overline{S_{1}}}^{2}\right)^{*} \prec\left(S_{1}^{2}\right)^{*}=T \quad \text { and } \quad T=\overline{S_{1}^{2}} \prec{\overline{S_{1}}}^{2}
$$

which implies that $\bar{S}^{2}={\overline{S_{1}}}^{2}=T$.

Remark. Note that in the statement of Lemma 2.4, it follows directly from $\bar{S}^{2}=T$ that $\mathscr{D}(T) \subseteq \mathscr{D}(\bar{S})$.

Lemma 2.5. Let $P_{1}$ and $P_{2}$ be two symmetric operators on a complex Hilbert space $\mathscr{H}$ such that $\mathscr{D}\left(P_{1}\right)=\mathscr{D}\left(P_{2}\right)$. Let $\mathscr{L} \subseteq \mathscr{D}\left(P_{1}\right)$ be a dense linear subspace of $\mathscr{H}$ such that $\left.P_{1}\right|_{\mathscr{L}}=\left.P_{2}\right|_{\mathscr{L}}$. Assume that the latter operator is essentially self-adjoint. Then $P_{1}=P_{2}$.

Proof. Observe that

$$
\overline{P_{1} \mid \mathscr{L}} \prec \overline{P_{1}} \prec P_{1}^{*} \prec\left(P_{1} \mid \mathscr{L}\right)^{*}=\overline{P_{1} \mid \mathscr{L}}
$$

from which it follows that $\overline{P_{1}}=\overline{P_{1} \mid \mathscr{L}}$. Similarly, $\overline{P_{2}}=\overline{P_{2} \mid \mathscr{L}}$ and therefore $\overline{P_{1}}=\overline{P_{2}}$. It follows immediately that $P_{1}=P_{2}$.

\section{Smooth and analytic vectors of unitary representations}

Let $G$ be a Banach-Lie group, $\mathfrak{g}=\operatorname{Lie}(G)$, and $(\pi, \mathscr{H})$ be a unitary representation of $G$. For every $x \in \mathfrak{g}$, the skew-adjoint operator corresponding to the one-parameter unitary representation

$$
\mathbb{R} \rightarrow \mathrm{U}(\mathscr{H}), \quad t \mapsto \pi(\exp (t x))
$$

via Stone's theorem is denoted by $\mathrm{d} \pi(x)$. If $x=a+i b \in \mathfrak{g}^{\mathbb{C}}$, then we set

$$
\mathrm{d} \pi(x)=\mathrm{d} \pi(a)+i \mathrm{~d} \pi(b),
$$

where the right-hand side means the sum of two unbounded operators, that is,

$$
\mathscr{D}(\mathrm{d} \pi(x))=\mathscr{D}(\mathrm{d} \pi(a)) \cap \mathscr{D}(\mathrm{d} \pi(b)) .
$$


Recall that $\pi^{v}: G \mapsto \mathscr{H}$ denotes the orbit map defined in (4). Let $\mathscr{H}^{\infty}$ be the subspace of smooth vectors of $(\pi, \mathscr{H})$, that is,

$$
\mathscr{H}^{\infty}=\left\{v \in \mathscr{H}^{\infty}: \pi^{v} \text { is a smooth map }\right\} .
$$

If $\mathscr{H}^{\infty}$ is a dense subspace of $\mathscr{H}$ then the representation $(\pi, \mathscr{H})$ is called a smooth unitary representation.

As in [Neeb 2010a, Section 4], we endow the space $\mathscr{H}^{\infty}$ with the topology induced by the family of seminorms $\left\{\mathrm{q}_{n}\right\}_{n=0}^{\infty}$, where

$$
\mathrm{q}_{n}(v)=\sup \left\{\left\|\mathrm{d} \pi\left(x_{1}\right) \cdots \mathrm{d} \pi\left(x_{n}\right) v\right\|: x_{1}, \ldots, x_{n} \in \mathfrak{g},\left\|x_{1}\right\| \leq 1, \ldots,\left\|x_{n}\right\| \leq 1\right\} .
$$

With this topology $\mathscr{H}^{\infty}$ is a Fréchet space [Neeb 2010a, Proposition 5.4]. Moreover, the map

$$
\mathfrak{g} \times \mathscr{H}^{\infty} \rightarrow \mathscr{H}^{\infty}, \quad(x, v) \mapsto \mathrm{d} \pi(x) v
$$

is continuous [Neeb 2010a, Lemma 4.2] and the map

$$
G \times \mathscr{H}^{\infty} \rightarrow \mathscr{H}^{\infty}, \quad(g, v) \mapsto \pi(g) v
$$

is smooth [Neeb 2010a, Theorem 4.4].

A vector $v \in \mathscr{H}^{\infty}$ is called analytic if the orbit map $\pi^{v}: G \rightarrow \mathscr{H}$ is a real analytic function. The space of analytic vectors is denoted by $\mathscr{H}^{\omega}$. If $\mathscr{H}^{\omega}$ is a dense subspace of $\mathscr{H}$ then the representation $(\pi, \mathscr{H})$ is called an analytic unitary representation.

Proposition 3.1 below records well known facts about unitary representations of the real line and its proof is omitted.

Proposition 3.1. Let $(\pi, \mathscr{H})$ be a unitary representation of $\mathbb{R}$ and $A$ be the skewadjoint infinitesimal generator of $(\pi, \mathscr{H})$.

(i) A vector $v \in \mathscr{H}$ is smooth if and only if $v \in \mathscr{D}^{\infty}(A)$.

(ii) A vector $v \in \mathscr{H}$ is analytic if and only if $v \in \mathscr{D}^{\omega}(A)$.

(iii) Let $v \in \mathscr{D}^{\infty}(A)$ and $r>0$ be such that $\sum_{n=0}^{\infty} \frac{r^{n}}{n !}\left\|A^{n} v\right\|<\infty$. Then

$$
\pi(t) v=\sum_{n=0}^{\infty} \frac{t^{n}}{n !} A^{n} v \quad \text { for every } t \in(-r, r) .
$$

If $G$ is a Banach-Lie group and $\mathfrak{g}=\operatorname{Lie}(G)$ then for every $r>0$ we set

$$
B_{r}=\left\{x \in \mathfrak{g}^{\mathbb{C}}:\|x\|<r\right\} .
$$

Lemma 3.2. Let $G$ be a Banach-Lie group, $\mathfrak{g}=\operatorname{Lie}(G)$, $(\pi, \mathscr{H})$ be a unitary representation of $G$, and $v \in \mathscr{H}^{\infty}$. Then $v \in \mathscr{H}^{\omega}$ if and only if there exists an $r>0$ 
such that for every $x \in B_{r}$ the series

$$
f_{v}(x)=\sum_{n=0}^{\infty} \frac{1}{n !} \mathrm{d} \pi(x)^{n} v
$$

converges in $\mathscr{H}$ (and therefore defines an analytic map $B_{r} \rightarrow \mathscr{H}$ ).

Proof. Let $v \in \mathscr{H}^{\omega}$ and $x \in B_{r}$. By Proposition 3.1, for all sufficiently small $t>0$ we have

$$
\pi(\exp (t x)) v=\sum_{n=0}^{\infty} \frac{t^{n}}{n !} \mathrm{d} \pi(x)^{n} v .
$$

This means that the series (9) converges in an absorbing set. By [Neeb 2011, Lemma 4.4] for every integer $n \geq 0$ the function

$$
\mathfrak{g} \rightarrow \mathscr{H}, \quad x \mapsto \frac{1}{n !} \mathrm{d} \pi(x)^{n} v
$$

is a continuous homogeneous polynomial of degree $n$. Thus by Theorem C.1(i) the series (9) defines an analytic map in a neighborhood of zero in $\mathfrak{g}$. By Theorem C.2 this series also defines an analytic map in a neighborhood of zero in $\mathfrak{g}_{\mathbb{C}}$.

Conversely, assume the series (9) converges for every $x \in B_{r}$. By Theorem C.1(i) there exists an $r^{\prime}>0$ such that

$$
\sum_{n=0}^{\infty} \frac{1}{n !} \sup \left\{\left\|\mathrm{d} \pi(x)^{n} v\right\|: x \in B_{r^{\prime}}\right\}<\infty .
$$

Therefore Proposition 3.1(iii) implies that

$$
\pi^{v}(\exp (x))=f_{v}(x) \quad \text { for every } x \in B_{r^{\prime}} \cap \mathfrak{g} .
$$

From Theorem C.1(ii) it follows that $f_{v}$ is an analytic function in $B_{r}$. Therefore the orbit map $\pi^{v}$ is also analytic in a neighborhood of identity of $G$. It follows immediately that $v \in \mathscr{H}^{\omega}$.

Notation. For every $r>0$ set

$$
\mathscr{H}^{\omega, r}=\left\{v \in \mathscr{H}^{\infty}: \sum_{n=0}^{\infty} \frac{1}{n !} \mathrm{d} \pi(x)^{n} v \text { converges in } \mathscr{H} \text { for every } x \in B_{r}\right\} .
$$

From Lemma 3.2 it follows that $\mathscr{H}^{\omega}=\bigcup_{r>0} \mathscr{H}^{\omega, r}$, and Proposition 3.1(iii) shows that if $v \in \mathscr{H}^{\omega, r}$ then

$$
\pi(\exp (x)) v=f_{v}(x) \text { for every } x \in B_{r} \cap \mathfrak{g} .
$$


Lemma 3.3. Let $G$ be a Banach-Lie group, $(\pi, \mathscr{H})$ be a unitary representation of $G, v \in \mathscr{H}^{\infty}, r>0$, and $\mathfrak{g}=\operatorname{Lie}(G)$. Then $v \in \mathscr{H}^{\omega, r}$ if and only if the map $\left.\pi^{v} \circ \exp \right|_{B_{r} \cap \mathfrak{g}}$ extends to an analytic function $h_{v}: B_{r} \rightarrow \mathcal{H}$.

Proof. Let $v \in \mathscr{H}^{\omega, r}$. From Theorem C.1(ii) it follows that the series $f_{v}(x)$ of (9) defines an analytic function in $B_{r}$. By (11) we have

$$
\pi(\exp (x)) v=f_{v}(x) \quad \text { for every } x \in B_{r} \cap \mathfrak{g} .
$$

Therefore $\left.\pi^{v} \circ \exp \right|_{B_{r} \cap \mathfrak{g}}$ extends to an analytic function in $B_{r}$.

Conversely, assume that $\left.\pi^{v} \circ \exp \right|_{B_{r} \cap \mathfrak{g}}$ extends to an analytic map $h_{v}: B_{r} \rightarrow \mathscr{H}$. Since $B_{r}$ is a balanced neighborhood of zero, from Theorem C. 4 it follows that

$$
h_{v}(x)=\sum_{n=0}^{\infty} \frac{1}{n !} \delta_{0}^{(n)} h_{v}(x) \quad \text { for every } x \in B_{r} .
$$

By Theorem C.4(i), for every $n \geq 0$ the function $\delta_{0}^{(n)} h_{v}: \mathfrak{g}^{\mathbb{C}} \rightarrow \mathscr{H}$ is a continuous homogeneous polynomial of degree $n$. Observe that $h_{v}(x)=\pi(\exp (x)) v$ for every $x \in B_{r} \cap \mathfrak{g}$, and by taking the $n$-th directional derivatives of both sides we obtain

$$
\delta_{0}^{(n)} h_{v}(x)=\mathrm{d} \pi(x)^{n} v \quad \text { for every } x \in B_{r} \cap \mathfrak{g} .
$$

Both sides of (12) are continuous homogeneous polynomials, and in particular analytic in $B_{r}$. Therefore by analytic continuation, the equality (12) holds for every $x \in B_{r}$. Consequently, the series (9) converges for every $x \in B_{r}$, that is, $v \in \mathscr{H}^{\omega, r}$.

Lemma 3.4. Let $G$ be a Banach-Lie group and $\mathfrak{g}=\operatorname{Lie}(G)$. Then there exists an $r_{\circ}>0$ such that for every $0<r<r_{\circ}$, every unitary representation $(\pi, \mathscr{H})$ of $G$, and every $v \in \mathscr{H}^{\omega, r}$, the following statements hold.

(i) $f_{v}(x) \in \mathscr{H}^{\omega}$ for every $x \in B_{r}$.

(ii) If $a \in \mathfrak{g}^{\mathbb{C}}$ then the map

$$
u_{a}: B_{r} \rightarrow \mathscr{H}, \quad u_{a}(x)=\mathrm{d} \pi(a)\left(f_{v}(x)\right)
$$

is analytic in $B_{r}$.

Proof. (i) Let $z \star z^{\prime}$ denote the Baker-Campbell-Hausdorff series for two elements $z, z^{\prime} \in \mathfrak{g}^{\mathbb{C}}$ whenever it converges. Choose $r_{\circ}>0$ small enough that the map

$$
\mu: B_{r_{\circ}} \times B_{r_{\circ}} \rightarrow \mathfrak{g}^{\mathbb{C}}, \quad \mu\left(z, z^{\prime}\right)=z \star z^{\prime}
$$

is analytic in $B_{r_{\circ}} \times B_{r_{0}}$.

Let $x \in B_{r}$. We write $x=x^{\prime}+i x^{\prime \prime}$, where $x^{\prime}, x^{\prime \prime} \in \mathfrak{g}$, and consider the complex subspace $\mathscr{V}=\operatorname{Span}_{\mathbb{C}}\left\{x^{\prime}, x^{\prime \prime}\right\}$ of $\mathfrak{g}^{\mathbb{C}}$. Choose $s>0$ such that $\|x\|<s<r$, and let 
$\overline{B_{s}}$ denote the closure of $B_{s}$. Since $\mathscr{V}$ is finite-dimensional, $\overline{B_{S}} \cap \mathcal{V}$ is a compact subset of $W$. It follows that there exists an $0<r^{\prime}<r$ such that

$$
\left\{z \star z^{\prime}: z \in B_{r^{\prime}} \quad \text { and } \quad z^{\prime} \in B_{s} \cap \mathscr{V}\right\} \subseteq B_{r} .
$$

Consequently, for every $z \in B_{r^{\prime}}$ the map

$$
\phi_{z}: B_{s} \cap \mathscr{V} \rightarrow \mathscr{H}, \quad \phi_{z}(y)=f_{v}(z \star y)
$$

is well-defined and analytic.

Next fix $z \in B_{r^{\prime}} \cap \mathfrak{g}$ and consider the function

$$
\psi_{z}: B_{s} \rightarrow \mathscr{H}, \quad \psi_{z}(y)=\pi(\exp (z)) f_{v}(y)
$$

which is analytic in $B_{s}$. If $y \in B_{s} \cap \mathscr{V} \cap \mathfrak{g}$, then $z \star y \in B_{r} \cap \mathfrak{g}$ and by (11) we have

$$
\phi_{z}(y)=f_{v}(z \star y)=\pi(\exp (z \star y)) v=\pi(\exp (z)) \pi(\exp (y)) v=\psi_{z}(y) .
$$

As both $\phi_{z}$ and $\psi_{z}$ are analytic in $B_{s} \cap \mathcal{V}$, it follows that the equality $\phi_{z}(y)=\psi_{z}(y)$ holds for every $y \in B_{s} \cap \mathscr{V}$. In particular, for every $z \in B_{r^{\prime}} \cap \mathfrak{g}$ we have

$$
f_{v}(z \star x)=\pi(\exp (z)) f_{v}(x) .
$$

This implies that the map

$$
G \rightarrow \mathscr{H}, \quad g \mapsto \pi(g) f_{v}(x)
$$

is analytic in a neighborhood of the identity, that is, $f_{v}(x) \in \mathscr{H}^{\omega}$. This completes the proof of (i).

(ii) It suffices to prove the statement when $a \in \mathfrak{g}$. If $0<r<r_{\circ}$ then there exists an open set $W \subseteq B_{r} \times B_{r}$ such that $\{0\} \times B_{r} \subseteq W$ and for every $\left(z, z^{\prime}\right) \in W$ we have $z \star z^{\prime} \in B_{r}$. Observe that the map

$$
\Psi: W \rightarrow \mathscr{H}, \quad \Psi\left(z, z^{\prime}\right)=f_{v}\left(z \star z^{\prime}\right)
$$

is analytic in $W$. The map

$$
\mathbb{C} \rightarrow \mathscr{H}, \quad \zeta \mapsto f_{v}((\zeta \cdot a) \star x)
$$

is an analytic function of $\zeta$ in a neighborhood of the origin. From (13) it follows that for every $x \in B_{r}$ we have

$$
\mathrm{d} \pi(a)\left(f_{v}(x)\right)=\left.\frac{\partial}{\partial \zeta} f_{v}((\zeta \cdot a) \star x)\right|_{\zeta=0} .
$$

From analyticity of the map $\Psi: W \rightarrow \mathscr{H}$ defined in (14) it follows that the map

$$
B_{r} \rightarrow \mathscr{H},\left.\quad x \mapsto \frac{\partial}{\partial \zeta} f_{v}((\zeta \cdot a) \star x)\right|_{\zeta=0}
$$

is analytic in $B_{r}$. This completes the proof of (ii). 


\section{Representations of Banach-Lie supergroups}

Our next task is to define the notions of smooth and analytic unitary representations of a Banach-Lie supergroup. The definitions are similar to the one given in [Carmeli et al. 2006, Definition 2] for finite-dimensional Lie supergroups.

Definition 4.1. Let $(G, \mathfrak{g})$ be a Banach-Lie supergroup. A smooth unitary representation (respectively, an analytic unitary representation) of $(G, \mathfrak{g})$ is a triple $\left(\pi, \rho^{\pi}, \mathscr{H}\right)$ satisfying the following properties.

(i) $(\pi, \mathscr{H})$ is a smooth (respectively, analytic) unitary representation of $G$ on the $\mathbb{Z}_{2}$-graded Hilbert space $\mathscr{H}$ such that for every $g \in G$, the operator $\pi(g)$ preserves the $\mathbb{Z}_{2}$-grading.

(ii) $\rho^{\pi}: \mathfrak{g} \rightarrow \operatorname{End}_{\mathbb{C}}(\mathscr{B})$ is a representation of the Banach-Lie superalgebra $\mathfrak{g}$, where $\mathscr{B}=\mathscr{H}^{\infty}$ (respectively, $\mathscr{B}=\mathscr{H}^{\omega}$ ).

(iii) $\rho^{\pi}(x)=\left.\mathrm{d} \pi(x)\right|_{\Re}$ for every $x \in \mathfrak{g}_{\overline{0}}$.

(iv) $e^{-\pi i / 4} \rho^{\pi}(x)$ is a symmetric operator for every $x \in \mathfrak{g}_{\overline{1}}$.

(v) Every element of the component group $G / G^{\circ}$ has a coset representative $g \in G$ such that $\pi(g) \rho^{\pi}(x) \pi(g)^{-1}=\rho^{\pi}(\operatorname{Ad}(g) x)$ for every $x \in \mathfrak{g}_{\overline{1}}$.

The category of smooth (respectively, analytic) unitary representations of $(G, \mathfrak{g})$ is denoted by $\operatorname{Rep}^{\infty}(G, \mathfrak{g})$ (respectively, $\operatorname{Rep}^{\omega}(G, \mathfrak{g})$ ).

Remark. Observe that in Definition 4.1(ii) the map $\rho^{\pi}$ is an abstract representation, that is, there are no continuity assumptions. If $G$ is connected then obviously Definition 4.1(v) always holds trivially. This point is the main difference between Definition 4.1 above and the definition given in [Carmeli et al. 2006, Definition 2] for finite-dimensional Lie groups, where it is assumed that

$$
\pi(g) \rho^{\pi}(x) \pi(g)^{-1}=\rho^{\pi}(\operatorname{Ad}(g) x) \text { for every } x \in \mathfrak{g}_{\overline{1}} \text { and every } g \in G,
$$

while the infinitesimal action is supposed to satisfy a weaker condition. Indeed Proposition 4.8 below implies that, for a (possibly disconnected) $G$, Equation (15) follows from Definition 4.1.

We will need a slightly more general gadget than smooth and analytic unitary representations, and we introduce it in the next definition.

Definition 4.2. Let $(G, \mathfrak{g})$ be a Banach-Lie supergroup. A prerepresentation of $(G, \mathfrak{g})$ is a 4-tuple $\left(\pi, \mathscr{H}, \mathscr{B}, \rho^{\mathscr{B}}\right)$ which satisfies the following properties.

(i) $(\pi, \mathscr{H})$ is a unitary representation of $G$ on the $\mathbb{Z}_{2}$-graded Hilbert space $\mathscr{H}=$ $\mathscr{H}_{\overline{0}} \oplus \mathscr{H}_{\overline{1}}$. Moreover, $\pi(g)$ is an even operator for every $g \in G$. 
(ii) $\mathscr{B}$ is a dense $\mathbb{Z}_{2}$-graded subspace of $\mathscr{H}$ such that

$$
\mathscr{B} \subseteq \bigcap_{x \in \mathfrak{g}_{\overline{0}}} \mathscr{D}(\mathrm{d} \pi(x)) .
$$

(iii) $\rho^{\mathscr{B}}: \mathfrak{g} \rightarrow \operatorname{End}_{\mathbb{C}}(\mathscr{B})$ is a representation of the Banach-Lie superalgebra $\mathfrak{g}$.

(iv) If $x \in \mathfrak{g}_{0}$ then $\rho^{\mathscr{R}}(x)=\left.\mathrm{d} \pi(x)\right|_{\mathscr{B}}$ and $\rho^{\mathscr{B}}(x)$ is essentially skew-adjoint.

(v) If $x \in \mathfrak{g}_{\overline{1}}$ then $e^{-\pi i / 4} \rho^{\mathscr{R}}(x)$ is a symmetric operator.

(vi) For every element of the component group $G / G^{\circ}$, there exists a coset representative $g \in G$ such that $\pi(g)^{-1} \mathscr{B} \subseteq \mathscr{B}$ and

$$
\pi(g) \rho^{\mathscr{R}}(x) \pi(g)^{-1}=\rho^{\mathscr{B}}(\operatorname{Ad}(g) x) \quad \text { for every } x \in \mathfrak{g}_{\overline{1}} .
$$

Remark. (i) Observe that in Definition 4.2(iii) there are no continuity assumptions on the map $\rho^{\mathscr{B}}$.

(ii) Definition 4.2 implies that $\mathscr{B} \subseteq \bigcap_{n \in \mathbb{N}} \mathscr{D}_{n}$, where

$$
\mathscr{D}_{n}=\bigcap_{x_{1}, \ldots, x_{n} \in \mathfrak{g}_{\overline{0}}} \mathscr{D}\left(\mathrm{d} \pi\left(x_{1}\right) \cdots \mathrm{d} \pi\left(x_{n}\right)\right) .
$$

Consequently, it follows from [Neeb 2010a, Theorem 9.4] that $\mathscr{B} \subseteq \mathscr{H}^{\infty}$. Since $\mathscr{B}$ is assumed to be dense in $\mathscr{H}$, the unitary representation $(\pi, \mathscr{H})$ of $G$ is smooth.

(iii) When $G$ is connected, Definition 4.2(vi) always holds trivially. The advantage of assuming the conjugacy invariance only for coset representatives (and not for every element of $G$ ) is that Theorem 4.10 will be applicable to the situations where $\mathscr{B}$ is not $G$-invariant. An example of this situation is the Fock space realization of the oscillator representation of $\left(\mathrm{OSp}_{\text {res }}(\mathscr{K}), \widehat{\mathfrak{o s p}}_{\text {res }}(\mathscr{K})\right)$. See Section 5 for details.

For a Banach-Lie group $G$, the subspaces of smooth and analytic vectors of a unitary representation are $G$-invariant. Therefore Lemma 2.2 has the following immediate consequence.

Corollary 4.3. Let $(G, \mathfrak{g})$ be a Banach-Lie supergroup, $\left(\pi, \rho^{\pi}, \mathcal{H}\right)$ be a smooth (respectively, analytic) unitary representation of $\left(G, \mathfrak{g}\right.$ ), and $\mathscr{P}=\mathscr{H}^{\infty}$ (respectively, $\left.\mathscr{B}=\mathscr{H}^{\omega}\right)$. Then $\left(\pi, \mathscr{H}, \mathscr{B}, \rho^{\pi}\right)$ is a prerepresentation of $(G, \mathfrak{g})$.

Lemma 4.4. Let $(G, \mathfrak{g})$ be a Banach-Lie supergroup and $\left(\pi, \mathscr{H}, \mathscr{B}, \rho^{\mathscr{B}}\right)$ be a prerepresentation of $(G, \mathfrak{g})$. Then the following statements hold.

(i) For every $x \in \mathfrak{g}_{\overline{0}}$ we have $\overline{\rho^{\mathscr{P}}(x)}=\mathrm{d} \pi(x)$. In particular $\mathscr{H}^{\infty} \subseteq \mathscr{D}\left(\overline{\rho^{\mathscr{P}}(x)}\right)$.

(ii) For every $x \in \mathfrak{g}_{1}$ the operator $e^{-\pi i / 4} \rho^{\mathscr{B}}(x)$ is essentially self-adjoint and $\left(\overline{\rho^{\mathscr{P}}(x)}\right)^{2}=\frac{1}{2} \mathrm{~d} \pi([x, x])$. In particular $\mathscr{H}^{\infty} \subseteq \mathscr{D}\left(\overline{\rho^{\mathscr{P}}(x)}\right)$.

Proof. (i) The statement follows directly from Definition 4.2(iv).

(ii) The statement follows from Lemma 2.4. 
Notation. Let $\left(\pi, \mathscr{H}, \mathscr{B}, \rho^{\mathscr{R}}\right)$ be a prerepresentation of a Banach-Lie supergroup $(G, \mathfrak{g})$. For every $x=x_{\overline{0}}+x_{\overline{1}} \in \mathfrak{g}^{\mathbb{C}}$ we define a linear operator $\tilde{\rho}^{\mathscr{P}_{3}}(x)$ on $\mathscr{H}$ with $\mathscr{D}\left(\tilde{\rho}^{\mathscr{P}}(x)\right)=\mathscr{H}^{\infty}$ as follows. If $x_{\overline{0}}=a_{\overline{0}}+i b_{\overline{0}}$ and $x_{\overline{1}}=a_{\overline{1}}+i b_{\overline{1}}$, where $a_{\overline{0}}, b_{\overline{0}} \in \mathfrak{g}_{\overline{0}}$ and $a_{\overline{1}}, b_{\overline{1}} \in \mathfrak{g}_{\overline{1}}$, then for every $v \in \mathscr{H}^{\infty}$ we set

$$
\tilde{\rho}^{\mathscr{P}}(x) v=\overline{\rho^{\mathscr{P}}\left(a_{\overline{0}}\right)} v+i \overline{\rho^{\mathscr{P}}\left(b_{\overline{0}}\right)} v+\overline{\rho^{\mathscr{P}}\left(a_{\overline{1}}\right)} v+i \overline{\rho^{\mathscr{R}}\left(b_{\overline{1}}\right)} v .
$$

Proposition 4.5. Let $(G, \mathfrak{g})$ be a Banach-Lie supergroup and $\left(\pi, \mathscr{H}, \mathscr{B}, \rho^{\mathscr{B}}\right)$ be a prerepresentation of $(G, \mathfrak{g})$. Then $\tilde{\rho}^{\mathscr{B}}(x) \mathscr{H}^{\infty} \subseteq \mathscr{H}^{\infty}$ for every $x \in \mathfrak{g}^{\mathbb{C}}$.

Proof. By Lemma 4.4(i) and the definition of $\tilde{\rho}^{\mathscr{P}}$ it suffices to prove the statement when $x \in \mathfrak{g}_{\overline{1}}$. As shown in [Neeb 2010a, Theorem 9.4], we have $\mathscr{H}^{\infty}=\bigcap_{n \in \mathbb{N}} \mathscr{D}_{n}$, where

$$
\mathscr{D}_{n}=\bigcap_{x_{1}, \ldots, x_{n} \in \mathfrak{g}_{\overline{0}}} \mathscr{D}\left(\mathrm{d} \pi\left(x_{1}\right) \cdots \mathrm{d} \pi\left(x_{n}\right)\right) .
$$

Therefore it is enough to prove that

$$
\overline{\rho^{\mathscr{R}}(x)} v \in \mathscr{D}_{n} \quad \text { for every } x \in \mathfrak{g}_{\overline{1}} \text { and } v \in \mathscr{H}^{\infty} .
$$

Let $y \in \mathfrak{g}_{\overline{0}}$. For every $w \in \mathscr{B}$, using Lemma 4.4 we can write

$$
\begin{aligned}
\left\langle\overline{\rho^{\mathscr{P}}(x)} v, \mathrm{~d} \pi(y) w\right\rangle & =\left\langle\overline{\rho^{\mathscr{R}}(x)} v, \rho^{\mathscr{R}}(y) w\right\rangle \\
& =e^{\pi i / 2}\left\langle v, \overline{\rho^{\mathscr{P}}(x)} \rho^{\mathscr{R}}(y) w\right\rangle \\
& =e^{\pi i / 2}\left\langle v, \rho^{\mathscr{R}}(y) \rho^{\mathscr{R}}(x) w+\rho^{\mathscr{B}}([x, y]) w\right\rangle \\
& =e^{\pi i / 2}\left\langle v, \rho^{\mathscr{P}}(y) \rho^{\mathscr{R}}(x) w\right\rangle+e^{\pi i / 2}\left\langle v, \rho^{\mathscr{R}}([x, y]) w\right\rangle \\
& =\left\langle\overline{\rho^{\mathscr{R}}(x)} \mathrm{d} \pi(y) v, w\right\rangle+\left\langle\overline{\rho^{\mathscr{R}}([x, y])} v, w\right\rangle .
\end{aligned}
$$

It follows that the $\mathbb{C}$-linear functional

$$
\mathscr{B} \rightarrow \mathbb{C}, \quad w \mapsto\left\langle\overline{\rho^{\mathscr{P}}(x)} v, \mathrm{~d} \pi(y) w\right\rangle
$$

is continuous, that is, $\overline{\rho^{\mathscr{B}}(x)} v \in \mathscr{D}\left(\left(\left.\mathrm{d} \pi(y)\right|_{\mathscr{B}}\right)^{*}\right)$. Since $\left.\mathrm{d} \pi(y)\right|_{\mathscr{B}}=\rho^{\mathscr{R}}(y)$ is essentially skew-adjoint, from Lemma 4.4(i) it follows that $\left(\left.\mathrm{d} \pi(y)\right|_{\mathscr{B}}\right)^{*}=-\mathrm{d} \pi(y)$, that is, $\overline{\rho^{\mathscr{B}}(x)} v \in \mathscr{D}(\mathrm{d} \pi(y))$. This proves (17) for $n=1$.

For $n>1$ the proof of (17) can be completed by induction. Let $x_{1}, \ldots, x_{n} \in \mathfrak{g}_{\overline{0}}$ and $v \in \mathscr{H}^{\infty}$. Using the induction hypothesis, for every $w \in \mathscr{B}$ we can write

$$
\begin{aligned}
& \left\langle\mathrm{d} \pi\left(x_{n-1}\right) \cdots \mathrm{d} \pi\left(x_{1}\right) \overline{\rho^{\mathscr{R}}(x)} v, \mathrm{~d} \pi\left(x_{n}\right) w\right\rangle=e^{\left(n-\frac{1}{2}\right) \pi i}\left\langle v, \rho^{\mathscr{R}}(x) \rho^{\mathscr{P}}\left(x_{1}\right) \cdots \rho^{\mathscr{R}}\left(x_{n}\right) w\right\rangle \\
& =e^{\left(n-\frac{1}{2}\right) \pi i}\left\langle v, \rho^{\mathscr{R}}\left(\left[x, x_{1}\right]\right) \rho^{\mathscr{R}}\left(x_{2}\right) \cdots \rho^{\mathscr{R}}\left(x_{n}\right) w+\rho^{\mathscr{R}}\left(x_{1}\right) \rho^{\mathscr{P}}(x) \rho^{\mathscr{R}}\left(x_{2}\right) \cdots \rho^{\mathscr{B}}\left(x_{n}\right) w\right\rangle \\
& =\left\langle\mathrm{d} \pi\left(x_{n}\right) \cdots \mathrm{d} \pi\left(x_{2}\right) \overline{\rho^{\mathscr{P}}\left(\left[x, x_{1}\right]\right)} v, w\right\rangle+\left\langle\mathrm{d} \pi\left(x_{n}\right) \cdots \mathrm{d} \pi\left(x_{2}\right) \overline{\rho^{\mathscr{R}}(x)} \mathrm{d} \pi\left(x_{1}\right) v, w\right\rangle .
\end{aligned}
$$


An argument similar to the case $n=1$ proves that

$$
\mathrm{d} \pi\left(x_{n-1}\right) \cdots \mathrm{d} \pi\left(x_{1}\right) \overline{\rho^{\mathscr{R}}(x)} v \in \mathscr{D}\left(\mathrm{d} \pi\left(x_{n}\right)\right) .
$$

Consequently, $v \in \mathscr{D}_{n}$.

Proposition 4.6. Let $(G, \mathfrak{g})$ be a Banach-Lie supergroup and $\left(\pi, \mathscr{H}, \mathscr{B}, \rho^{\mathscr{R}}\right)$ be a prerepresentation of $(G, \mathfrak{g})$. Then the following statements hold.

(i) The map

$$
\mathfrak{g}^{\mathbb{C}} \times \mathscr{H}^{\infty} \rightarrow \mathscr{H}^{\infty}, \quad(x, v) \mapsto \tilde{\rho}^{\mathscr{P}}(x) v
$$

is $\mathbb{C}$-bilinear.

(ii) If $x, y \in \mathfrak{g}^{\mathbb{C}}$ are homogeneous, then for every $v \in \mathscr{H}^{\infty}$ we have

$$
\tilde{\rho}^{\mathscr{R}}([x, y]) v=\tilde{\rho}^{\Re_{R}}(x) \tilde{\rho}^{\mathscr{R}}(y) v-(-1)^{p(x) p(y)} \tilde{\rho}^{\mathscr{\Re}}(y) \tilde{\rho}^{\mathscr{P}_{R}}(x) v .
$$

(iii) The map given in (18) is continuous.

Proof. (i) By Lemma 4.4(i) and the definition of $\tilde{\rho}^{\mathscr{B}}$ it is enough to prove that for every $v \in \mathscr{H}^{\infty}$ the map

$$
\mathfrak{g}_{\overline{1}} \rightarrow \mathscr{H}^{\infty}, \quad x \mapsto \overline{\rho^{\mathscr{P}}(x)} v
$$

is $\mathbb{R}$-linear.

Let $x \in \mathfrak{g}_{\overline{1}}$ and $a \in \mathbb{R}$. Then the equality

$$
\overline{\rho^{\mathscr{R}}(a x)} v=a \overline{\rho^{\mathscr{P}}(x)} v
$$

holds for every $v \in \mathscr{B}$, and therefore by Lemma 2.5 it also holds for every $v \in \mathscr{H}^{\infty}$. A similar reasoning proves that if $x, y \in \mathfrak{g}_{1}$ then for every $v \in \mathscr{H}^{\infty}$ we have

$$
\overline{\rho^{\mathscr{P}}(x+y)} v=\overline{\rho^{\mathscr{R}}(x)} v+\overline{\rho^{\mathscr{R}}(y)} v .
$$

(ii) It suffices to prove the statement for $x, y \in \mathfrak{g}$. Depending on the parities of $x$ an $y$, there are four cases to consider, but the argument for all of them is essentially the same. For example, if $x \in \mathfrak{g}_{\overline{0}}$ and $y \in \mathfrak{g}_{\overline{1}}$, then we define two operators $P_{1}$ and $P_{2}$ with domains $\mathscr{D}\left(P_{1}\right)=\mathscr{D}\left(P_{2}\right)=\mathscr{H}^{\infty}$ as follows. If $v \in \mathscr{H}^{\infty}$ then we set $P_{1} v=e^{-\pi i / 4} \overline{\rho^{\mathscr{R}}([x, y])} v \quad$ and $\quad P_{2} v=e^{-\pi i / 4}\left(\overline{\rho^{\mathscr{R}}(x)} \overline{\rho^{\mathscr{R}}(y)} v-\overline{\rho^{\mathscr{R}}(y)} \overline{\rho^{\mathscr{R}}(x)} v\right)$.

Then $P_{1}$ and $P_{2}$ are both symmetric, $\left.P_{1}\right|_{\mathscr{B}}=\left.P_{2}\right|_{\mathscr{B}}$, and by Lemma 4.4(ii) the operator $\left.P_{1}\right|_{\Re}$ is essentially self-adjoint. Lemma 2.5 implies that $P_{1}=P_{2}$.

(iii) As in (i), it is enough to prove that the map

$$
\mathfrak{g}_{\overline{1}} \times \mathscr{H}^{\infty} \rightarrow \mathscr{H}^{\infty}, \quad x \mapsto \overline{\rho^{\mathscr{P}}(x)} v
$$


is continuous. Let $v \in \mathscr{H}^{\infty}$ and $y \in \mathfrak{g}_{\overline{1}}$. Setting $P_{1}=e^{-\pi i / 4} \overline{\rho^{\mathscr{P}}(y)}$ and $P_{2}=$ $\frac{1}{2} e^{-\pi i / 2} \mathrm{~d} \pi([y, y])$ in Lemma 2.3 we obtain

$$
\begin{aligned}
\left\|\overline{\rho^{\mathscr{R}}(y)} v\right\| & \leq \frac{1}{\sqrt{2}}\|v\|^{\frac{1}{2}} \cdot\|\mathrm{d} \pi([y, y]) v\|^{\frac{1}{2}} \\
& \leq \frac{1}{\sqrt{2}} \mathrm{q}_{0}(v)^{\frac{1}{2}} \mathrm{q}_{1}(v)^{\frac{1}{2}} \cdot\|[y, y]\|^{\frac{1}{2}} \leq \frac{1}{2 \sqrt{2}}\left(\mathrm{q}_{0}(v)+\mathrm{q}_{1}(v)\right) \cdot\|y\|
\end{aligned}
$$

from which it follows that

$$
\mathrm{q}_{0}\left(\overline{\rho^{\mathscr{P}}(y)} v\right) \leq \frac{1}{2 \sqrt{2}}\left(\mathrm{q}_{0}(v)+\mathrm{q}_{1}(v)\right) \cdot\|y\| .
$$

If $x \in \mathfrak{g}_{0}$ satisfies $\|x\| \leq 1$ then $\|[x, y]\| \leq\|y\|$ and using (22) we obtain

$$
\begin{aligned}
\| \mathrm{d} \pi(x) & \overline{\rho^{\mathscr{P}}(y)} v \| \\
& =\mathrm{q}_{0}\left(\overline{\rho^{\mathscr{P}}(x)} \overline{\rho^{\mathscr{P}}(y)} v\right) \leq \mathrm{q}_{0}\left(\overline{\rho^{\mathscr{P}}(y)} \overline{\rho^{\mathscr{P}}(x)} v\right)+\mathrm{q}_{0}\left(\overline{\rho^{\mathscr{P}}([x, y])} v\right) \\
& \leq \frac{1}{2 \sqrt{2}}\left(\mathrm{q}_{0}(\mathrm{~d} \pi(x) v)+\mathrm{q}_{1}(\mathrm{~d} \pi(x) v)\right) \cdot\|y\|+\frac{1}{2 \sqrt{2}}\left(\mathrm{q}_{0}(v)+\mathrm{q}_{1}(v)\right) \cdot\|[x, y]\| \\
& \leq \frac{1}{2 \sqrt{2}}\left(\mathrm{q}_{1}(v)+\mathrm{q}_{2}(v)\right) \cdot\|y\|+\frac{1}{2 \sqrt{2}}\left(\mathrm{q}_{0}(v)+\mathrm{q}_{1}(v)\right) \cdot\|y\| \\
& =\frac{1}{2 \sqrt{2}}\left(\mathrm{q}_{0}(v)+2 \mathrm{q}_{1}(v)+\mathrm{q}_{2}(v)\right) \cdot\|y\|,
\end{aligned}
$$

from which it follows that

$$
\mathrm{q}_{1}\left(\overline{\rho^{\mathscr{B}}(y)} v\right) \leq \frac{1}{2 \sqrt{2}}\left(\mathrm{q}_{0}(v)+2 \mathrm{q}_{1}(v)+\mathrm{q}_{2}(v)\right) \cdot\|y\| .
$$

More generally, if $x_{1}, \ldots, x_{n} \in \mathfrak{g}_{0}$ satisfy $\left\|x_{1}\right\| \leq 1, \ldots,\left\|x_{n}\right\| \leq 1$, we can use the equality

$$
\begin{aligned}
& \mathrm{d} \pi\left(x_{1}\right) \cdots \mathrm{d} \pi\left(x_{n}\right) \overline{\rho^{\mathscr{R}}(y)} v=\overline{\rho^{\mathscr{P}}(y)} \overline{\rho^{\mathscr{P}}\left(x_{1}\right)} \cdots \overline{\rho^{\mathscr{P}}\left(x_{n}\right)} v \\
& \quad+\sum_{i=1}^{n} \overline{\rho^{\mathscr{P}}\left(x_{1}\right)} \cdots \overline{\rho^{\mathscr{P}}\left(x_{i-1}\right)} \overline{\rho^{\mathscr{R}}\left(\left[x_{i}, y\right]\right)} \overline{\rho^{\mathscr{R}}\left(x_{i+1}\right)} \cdots \overline{\rho^{\mathscr{R}}\left(x_{n}\right)} v
\end{aligned}
$$

to prove by induction on $n$ that

$$
\mathrm{q}_{n}\left(\rho^{\pi}(y) v\right) \leq \frac{1}{2 \sqrt{2}}\|y\| \cdot \sum_{k=0}^{n+1}\left(\begin{array}{c}
n+1 \\
k
\end{array}\right) \mathrm{q}_{k}(v) \quad \text { for every } n \geq 0 .
$$

From (24) the continuity of (21) follows immediately.

Our next goal is to prove Proposition 4.8 below. The proof of the latter proposition is based on a subtle lemma from [Jørgensen and Moore 1984, Chapter 3]. We use the lemma in the form given in [Merigon 2011]. 
Lemma 4.7. Let $A$ and $B$ be two linear operators on a complex Hilbert space $\mathscr{H}$ and $\mathscr{D}$ be a dense subspace of $\mathscr{H}$ with the following properties.

(i) $\mathscr{D}(A)=\mathscr{D}(B)=\mathscr{D}$.

(ii) A is essentially skew-adjoint.

(iii) $A \mathscr{D} \subseteq \mathscr{D}$.

(iv) $e^{t A} \mathscr{D} \subseteq \mathscr{D}$ for every $t \in \mathbb{R}$.

(v) B is closable.

Let $v \in \mathscr{D}$ be such that the map

$$
\mathbb{R} \rightarrow \mathscr{H}, \quad t \mapsto B A e^{t A} v
$$

is continuous. Then the map

$$
\mathbb{R} \rightarrow \mathscr{H}, \quad t \mapsto B e^{t A} v
$$

is differentiable and $\frac{d}{d t}\left(B e^{t A} v\right)=B A e^{t A} v$.

Proof. See [Merigon 2011, Lemma 5].

Proposition 4.8. Let $(G, \mathfrak{g})$ be a Banach-Lie supergroup where $G$ is connected and $\left(\pi, \mathscr{H}, \mathscr{B}, \rho^{\mathscr{R}}\right)$ be a prerepresentation of $(G, \mathfrak{g})$. Then for every $g \in G$, every $x \in \mathfrak{g}_{\overline{1}}^{\mathbb{C}}$, and every $v \in \mathscr{H}^{\infty}$ we have

$$
\pi(g) \tilde{\rho}^{\Re}(x) \pi(g)^{-1} v=\tilde{\rho}^{\Re}(\operatorname{Ad}(g) x) v .
$$

Proof. Fix $y \in \mathfrak{g}_{\overline{0}}$ and set $A(s)=\pi(\exp ((1-s) y))$ for every $s \in \mathbb{R}$. For every $v \in \mathscr{H}^{\infty}$ and every $s \in \mathbb{R}$ define

$$
K(s): \mathfrak{g}_{1}^{\mathbb{C}} \rightarrow \mathscr{H}, \quad K(s) x=A(s) \tilde{\rho}^{\mathscr{B}}(x) A(s)^{-1} v .
$$

If $g=\exp (y)$ and $\gamma(s)=\operatorname{Ad}(\exp (s y)) x$ for every $s \in \mathbb{R}$ then the left hand side of (25) is equal to $K(0) \gamma(0)$ and the right hand side of (25) is equal to $K(1) \gamma(1)$. Therefore it suffices to prove that the map $s \mapsto K(s) \gamma(s)$ is constant.

By Proposition 4.6, for every $t \in \mathbb{R}$ the operator $K(t)$ is bounded. From continuity of the maps (7) and (8) it follows that the map

$$
\mathbb{R} \rightarrow \mathscr{H}, \quad s \mapsto \tilde{\rho}^{\mathscr{P}}(x) \tilde{\rho}^{\mathscr{P}}(y) A(s)^{-1} v
$$

is continuous as well. It follows from Lemma 4.7 that the map

$$
\eta: \mathbb{R} \mapsto \mathscr{H}, \quad \eta(s)=\tilde{\rho}^{\mathscr{P}}(x) A(s)^{-1} v
$$

is differentiable, and $\eta^{\prime}(s)=\tilde{\rho}^{\mathscr{P}}(x) \tilde{\rho}^{\mathscr{R}}(y) A(s)^{-1} v$.

Next we show that the map

$$
\mathbb{R} \rightarrow \mathscr{H}, \quad s \mapsto K(s) x
$$


is differentiable and we compute its derivative. Observe that

$$
\frac{d}{d s}(K(s) x)=\frac{d}{d s}(A(s) \eta(s))=\lim _{h \rightarrow 0} \frac{1}{h}(A(s+h) \eta(s+h)-A(s) \eta(s))
$$

and

$$
\begin{aligned}
\frac{1}{h}(A(s+h) \eta(s+h)-A(s) \eta(s)) & \\
& =A(s+h)\left(\frac{1}{h}(\eta(s+h)-\eta(s))\right)+\frac{1}{h}(A(s+h) \eta(s)-A(s) \eta(s)) .
\end{aligned}
$$

The first term in (27) can be written as

$$
A(s+h)\left(\frac{1}{h}(\eta(s+h)-\eta(s))-\eta^{\prime}(s)\right)+A(s+h) \eta^{\prime}(s) .
$$

Since $\|A(s+h)\|_{\mathrm{Op}}=1$, when $h \rightarrow 0$ we obtain

$$
A(s+h)\left(\frac{1}{h}(\eta(s+h)-\eta(s))-\eta^{\prime}(s)\right) \rightarrow 0 \quad \text { and } \quad A(s+h) \eta^{\prime}(s) \rightarrow A(s) \eta^{\prime}(s) .
$$

Since $\eta(s) \in \mathscr{H}^{\infty}$, as $h \rightarrow 0$ the second term in (27) converges to $-A(s) \tilde{\rho}^{\mathscr{\Re}}(y) \eta(s)$. It follows that (28) $\frac{d}{d s}(K(s) x)=A(s) \eta^{\prime}(s)-A(s) \tilde{\rho}^{\Im_{\beta}}(y) \eta(s)=A(s)\left[\tilde{\rho}^{\mathscr{\beta}}(x), \tilde{\rho}^{\Im_{\beta}}(y)\right] A(s)^{-1} v$.

To complete the proof, it suffices to show that $\frac{d}{d s}(K(s) \gamma(s))=0$ for every $s \in \mathbb{R}$. We have

$$
\text { (29) } \begin{aligned}
\frac{1}{h}(K(s & +h) \gamma(s+h)-K(s) \gamma(s)) \\
& =K(s+h)\left(\frac{1}{h}(\gamma(s+h)-\gamma(s))\right)+\frac{1}{h}(K(s+h) \gamma(s)-K(s) \gamma(s)) .
\end{aligned}
$$

The first term in (29) can be written as

$$
K(s+h)\left(\frac{1}{h}(\gamma(s+h)-\gamma(s))-\gamma^{\prime}(s)\right)+K(s+h) \gamma^{\prime}(s)
$$

Differentiability of the map given in (26) implies that it is continuous, and in particular if $I$ is a compact interval containing $s$, then $\sup _{t \in I}\|K(t) x\|<\infty$. The Banach-Steinhaus theorem implies that $\sup _{t \in I}\|K(t)\|_{\mathrm{Op}}<\infty$. It follows that as $h \rightarrow 0$ the first term in (29) converges to

$$
K(s) \gamma^{\prime}(s)=A(s) \tilde{\rho}^{\mathscr{P}}([y, \gamma(s)]) A(s)^{-1} v .
$$

By (28), the second term in (29) converges to

$$
A(s)\left[\tilde{\rho}^{\mathscr{P}}(\gamma(s)), \tilde{\rho}^{\mathscr{R}}(y)\right] A(s)^{-1} v .
$$


It follows that

$$
\begin{aligned}
\frac{d}{d s}(K(s) \gamma(s)) & =A(s) \tilde{\rho}^{\mathscr{P}}([y, \gamma(s)]) A(s)^{-1} v+A(s)\left[\tilde{\rho}^{\mathscr{P}}(\gamma(s)), \tilde{\rho}^{\mathscr{\Re}}(y)\right] A(s)^{-1} v \\
& =0
\end{aligned}
$$

Proposition 4.9. Let $(G, \mathfrak{g})$ be a Banach-Lie supergroup and $\left(\pi, \mathscr{H}, \mathscr{B}, \rho^{\mathscr{B}}\right)$ be a prerepresentation of $(G, \mathfrak{g})$. Then there exists an $r_{\circ}>0$ such that for every positive $r<r_{\circ}$ and every $x \in \mathfrak{g}^{\mathbb{C}}$ we have $\tilde{\rho}^{\mathscr{\beta}}(x) \mathscr{H}^{\omega, r} \subseteq \mathscr{H}^{\omega, r}$. In particular, for every $x \in \mathfrak{g}^{\mathbb{C}}$ we have $\tilde{\rho}^{\mathscr{B}}(x) \mathscr{H}^{\omega} \subseteq \mathscr{H}^{\omega}$.

Proof. It suffices to prove the statement when $x$ is a homogeneous element of $\mathfrak{g}$. We give the argument for $x \in \mathfrak{g}_{\overline{1}}$. The argument for the case $x \in \mathfrak{g}_{\overline{0}}$ is analogous.

Let $r_{\circ}$ be the constant obtained from Lemma 3.4 and take $v \in \mathscr{H}^{\omega, r}$, where $0<r<r_{\circ}$. Recall that

$$
B_{r}=\left\{y \in \mathfrak{g}_{\overline{0}}^{\mathbb{C}}:\|y\|<r\right\} .
$$

The map $y \mapsto f_{v}(y)=\sum \frac{1}{n !} \mathrm{d} \pi(x)^{n} v$ of Lemma 3.2 is analytic in $B_{r}$. Since

$$
\sum_{n=0}^{\infty} \frac{1}{n !}\left\|\operatorname{ad}_{y}^{n}(x)\right\| \leq \sum_{n=0}^{\infty} \frac{1}{n !}\|y\|^{n} \cdot\|x\|<\infty,
$$

Theorem C.1(ii) implies that the map

$$
\mathfrak{g}_{\overline{0}}^{\mathbb{C}} \rightarrow \mathfrak{g}_{\overline{1}}^{\mathbb{C}}, \quad y \mapsto e^{\mathrm{ad}_{y}} x=\sum_{n=0}^{\infty} \frac{1}{n !} \operatorname{ad}_{y}^{n}(x)
$$

is analytic in $\mathfrak{g}_{0}^{\mathbb{C}}$.

Consider the map

$$
\varphi: \mathfrak{g}_{0}^{\mathbb{C}} \times B_{r} \rightarrow \mathscr{H}, \quad \varphi(y, z)=\tilde{\rho}^{\mathscr{P}}\left(e^{\mathrm{ad}_{y}} x\right) f_{v}(z) .
$$

Note that if $z \in B_{r}$ then by Lemma 3.4(i) we have $f_{v}(z) \in \mathscr{H}^{\omega}$ and therefore $\varphi$ is well-defined. Our next goal is to prove that $\varphi$ is separately analytic.

Fix $z \in B_{r}$ and let $w=f_{v}(z)$. We have $w \in \mathscr{H}^{\omega}$. Proposition 4.6 implies that the map

$$
\mathfrak{g}_{\overline{1}}^{\mathbb{C}} \rightarrow \mathscr{H}, \quad u \mapsto \tilde{\rho}^{\mathscr{P}}(u) w
$$

is $\mathbb{C}$-linear and continuous. Therefore analyticity of the map given in (30) implies that the map $y \mapsto \varphi(y, z)$ is analytic in $\mathfrak{g}_{\overline{0}}^{\mathbb{C}}$.

Next we prove analyticity in $B_{r}$ of the map $z \mapsto \varphi(y, z)$. Fix $y \in \mathfrak{g}_{\overline{0}}^{\mathbb{C}}$ and set $\tilde{y}=\sum_{n=0}^{\infty} \frac{1}{n !} \operatorname{ad}_{y}^{n}(x)$. Writing $\tilde{y}=a+i b$, where $a, b \in \mathfrak{g}_{1}$, and using $\mathbb{C}$-linearity of 
$\tilde{\rho}^{\mathscr{B}}$, it turns out that it suffices to prove analyticity in $B_{r}$ of the maps

$$
z \mapsto \tilde{\rho}^{\mathscr{R}}(a) f_{v}(z) \quad \text { and } \quad z \mapsto \tilde{\rho}^{\mathscr{R}}(b) f_{v}(z) .
$$

The argument for both cases is the same, and we only give it for the first case. Lemma 3.4(ii) implies that the map

$$
B_{r} \mapsto \mathscr{H}, \quad z \mapsto \tilde{\rho}^{\mathscr{S}}([a, a]) f_{v}(z)
$$

is analytic in $B_{r}$. The operator $T=e^{-\pi i / 4} \overline{\tilde{\rho}^{\mathscr{B}}(a)}$ is self-adjoint and spectral theory implies that $T\left(I+T^{2}\right)^{-1}$ is a bounded operator. Using Proposition 4.6(ii) we can write

$$
\begin{aligned}
\tilde{\rho}^{\Re}(a) f_{v}(z) & =e^{\pi i / 4} T\left(I+T^{2}\right)^{-1}\left(I+T^{2}\right) f_{v}(z) \\
& =e^{\pi i / 4} T\left(I+T^{2}\right)^{-1}\left(f_{v}(z)+\frac{1}{2} e^{-\pi i / 2} \tilde{\rho}^{\Re}([a, a]) f_{v}(z)\right),
\end{aligned}
$$

from which it follows that the map $z \mapsto \tilde{\rho}^{\mathscr{P}}(a) f_{v}(z)$ is analytic in $B_{r}$.

By Theorem C.3, the separately analytic map $\varphi(y, z)$ is analytic. Thus the map

$$
B_{r} \rightarrow \mathscr{H}, \quad y \mapsto \varphi(y, y)
$$

is analytic in $B_{r}$.

Let $y \in B_{r} \cap \mathfrak{g}_{0}$. By (11) we have $f_{v}(y)=\pi(\exp (y)) v$. Write $x=x^{\prime}+i x^{\prime \prime}$, where $x^{\prime}, x^{\prime \prime} \in \mathfrak{g}_{\overline{1}}$, and set $g=\exp (y)$. Using Proposition 4.8 we obtain

$$
\begin{aligned}
& \varphi(y, y)=\tilde{\rho}^{\mathscr{\Re}}\left(e^{\mathrm{ad}_{y}} x\right) f_{v}(y) \\
& =\tilde{\rho}^{\mathscr{B}}\left(\operatorname{Ad}(\exp (y)) x^{\prime}\right) f_{v}(y)+i \tilde{\rho}^{\mathscr{B}}\left(\operatorname{Ad}(\exp (y)) x^{\prime \prime}\right) f_{v}(y) \\
& =\pi(g) \tilde{\rho}^{\mathscr{B}}\left(x^{\prime}\right) \pi(g)^{-1} f_{v}(y)+i \pi(g) \tilde{\rho}^{\mathscr{R}}\left(x^{\prime \prime}\right) \pi(g)^{-1} f_{v}(y) \\
& =\pi(g) \tilde{\rho}^{\mathscr{P}_{3}}(x) \pi(g)^{-1} \pi(g) v=\pi(g) \tilde{\rho}^{\mathscr{\Re}}(x) v .
\end{aligned}
$$

From Lemma 3.3 it follows that $\tilde{\rho}^{\mathscr{B}}(x) v \in \mathscr{H}^{\omega, r}$.

We can now prove our first main theorem, which states that every prerepresentation of a Banach-Lie group corresponds to a unique unitary representation.

Theorem 4.10 (Stability theorem). Let $\left(\pi, \mathscr{H}, \mathscr{B}, \rho^{\mathscr{B}}\right)$ be a prerepresentation of a Banach-Lie supergroup $(G, \mathfrak{g})$.

(i) There exists a unique map

$$
\rho^{\pi}: \mathfrak{g} \rightarrow \operatorname{End}_{\mathbb{C}}\left(\mathscr{H}^{\infty}\right)
$$

such that $\left.\rho^{\pi}(x)\right|_{\mathscr{B}}=\rho^{\mathscr{B}}(x)$ and $\left(\pi, \rho^{\pi}, \mathscr{H}\right)$ is a smooth unitary representation of $(G, \mathfrak{g})$. 
(ii) If $(\pi, \mathscr{H})$ is an analytic unitary representation of $G$, then there exists a unique map

$$
\rho^{\pi}: \mathfrak{g} \rightarrow \operatorname{End}_{\mathbb{C}}\left(\mathscr{H}^{\omega}\right)
$$

such that $\left.\overline{\rho^{\pi}(x)}\right|_{\mathscr{B}}=\rho^{\mathscr{B}}(x)$ and $\left(\pi, \rho^{\pi}, \mathscr{H}\right)$ is an analytic unitary representation of $(G, \mathfrak{g})$.

Proof. (i) To prove the existence of $\rho^{\pi}$, we set $\rho^{\pi}(x)=\tilde{\rho}^{\mathscr{S}}(x)$ for every $x \in \mathfrak{g}$. Proposition 4.5 implies that $\tilde{\rho}^{\mathscr{P}}(x) \in \operatorname{End}_{\mathbb{C}}\left(\mathscr{H}^{\infty}\right)$. To prove the conjugacy invariance relation of Definition 4.1(v), for every element of $G / G^{\circ}$ we take a coset representative $g \in G$ which satisfies the condition of Definition 4.2(vi), and use Lemma 2.5 with $P_{1}=e^{-\pi i / 4} \pi(g) \tilde{\rho}^{\mathscr{P}}(x) \pi(g)^{-1}, P_{2}=e^{-\pi i / 4} \tilde{\rho}^{\mathscr{B}}(\operatorname{Ad}(g) x)$, and $\mathscr{L}=\mathscr{B}$.

To prove uniqueness, it suffices to show that if $\left(\pi, \rho^{\pi}, \mathscr{H}\right)$ is a smooth unitary representation such that for every $x \in \mathfrak{g}$ we have $\left.\rho^{\pi}(x)\right|_{\mathscr{B}}=\rho^{\mathscr{B}}(x)$ then for every $x \in \mathfrak{g}$ we have

$$
\left.\rho^{\pi}(x)\right|_{\mathscr{H}^{\infty}}=\tilde{\rho}^{\mathscr{P}}(x) .
$$

It suffices to prove (31) when $x$ is homogeneous. If $x \in \mathfrak{g}_{\overline{1}}$ then by Lemma 4.4(ii) the operator $\left.e^{-\pi i / 4} \tilde{\rho}^{\mathscr{P}_{B}}(x)\right|_{\Re}$ is essentially self-adjoint. Therefore (31) follows from setting $P_{1}=\left.e^{-\pi i / 4} \rho^{\pi}(x)\right|_{\mathscr{H}}, P_{2}=e^{-\pi i / 4} \tilde{\rho}^{\mathscr{B}}(x)$, and $\mathscr{L}=\mathscr{B}$ in Lemma 2.5. The argument for $x \in \mathfrak{g}_{0}$ is similar.

(ii) Existence follows from (i) and Proposition 4.9. The proof of uniqueness is similar to the one given in (i).

Let $\mathrm{F}: \operatorname{Rep}^{\omega}(G, \mathfrak{g}) \rightarrow \operatorname{Rep}^{\infty}(G, \mathfrak{g})$ be the functor defined by

$$
\left(\pi, \rho^{\pi}, \mathscr{H}\right) \mapsto\left(\pi, \tilde{\rho}^{\mathscr{H}}, \mathscr{H}\right) .
$$

A morphism between two objects of $\operatorname{Rep}^{\omega}(G, \mathfrak{g})$ will automatically become a morphism between their images under $\mathrm{F}$ in $\operatorname{Rep}^{\infty}(G, \mathfrak{g})$, and in fact $\mathrm{F}$ is fully faithful. Let $\operatorname{Rep}_{a}^{\infty}(G, \mathfrak{g})$ denote the full subcategory of $\operatorname{Rep}^{\infty}(G, \mathfrak{g})$ whose objects are smooth unitary representations $\left(\pi, \rho^{\pi}, \mathscr{H}\right)$ of $(G, \mathfrak{g})$ such that $(\pi, \mathscr{H})$ is an analytic unitary representation of $G$.

Corollary 4.11. The functor $\mathrm{F}$ is an isomorphism of the categories $\operatorname{Rep}^{\omega}(G, \mathfrak{g})$ and $\operatorname{Rep}_{a}^{\infty}(G, \mathfrak{g})$.

Proof. Immediate, from the uniqueness statement of Theorem 4.10(ii).

Remark. A natural question that arises from Corollary 4.11 is whether or not $\mathrm{F}$ is an isomorphism between the categories $\operatorname{Rep}^{\omega}(G, \mathfrak{g})$ and $\operatorname{Rep}^{\infty}(G, \mathfrak{g})$. We answer this question negatively in Appendix A by giving an example of a Banach-Lie group $G$ with a smooth unitary representation $(\pi, \mathscr{H})$ which does not have any analytic vectors. 
Let $(\phi, \varphi):(H, \mathfrak{h}) \rightarrow(G, \mathfrak{g})$ be a homomorphism of Banach-Lie supergroups. One can obtain restriction functors

$$
\operatorname{Res}^{\infty}: \operatorname{Rep}^{\infty}(G, \mathfrak{g}) \rightarrow \operatorname{Rep}^{\infty}(H, \mathfrak{h}) \quad \text { and } \operatorname{Res}^{\omega}: \operatorname{Rep}^{\omega}(G, \mathfrak{g}) \rightarrow \operatorname{Rep}^{\omega}(H, \mathfrak{h})
$$

associated to this homomorphism as follows. If $\left(\pi, \rho^{\pi}, \mathscr{H}\right)$ is a smooth (respectively, analytic) unitary representation of $(G, \mathfrak{g})$, then we set $\mathscr{L}=\mathscr{H}^{\infty}$ (respectively, $\left.\mathscr{L}=\mathscr{H}^{\omega}\right)$. Observe that $\left(\pi \circ \phi, \mathscr{H}, \mathscr{L}, \rho^{\mathscr{L}} \circ \varphi\right)$ is a prerepresentation of $(H, \mathfrak{h})$. The functor $\operatorname{Res}^{\infty}$ (respectively, $\operatorname{Res}^{\omega}$ ) maps $\left(\pi, \rho^{\pi}, \mathscr{H}\right.$ ) to the unique smooth (respectively, analytic) unitary representation of $(H, \mathfrak{h})$ which corresponds to this prerepresentation. (The existence and uniqueness of this unitary representation follows from Theorem 4.10.) In conclusion, we have proved the following theorem.

Theorem 4.12 (Restriction theorem). Let $(\phi, \varphi):(H, \mathfrak{h}) \rightarrow(G, \mathfrak{g})$ be a homomorphism of Banach-Lie supergroups, $\left(\pi, \mathscr{H}, \rho^{\pi}\right)$ be a smooth (respectively, analytic) unitary representation of $(G, \mathfrak{g})$, and $\mathscr{L}=\mathscr{H}^{\infty}$ (respectively, $\mathscr{L}=\mathscr{H}^{\omega}$ ). Then there exists a unique smooth (respectively, analytic) unitary representation $\left(\sigma, \mathscr{H}, \rho^{\sigma}\right)$ of $(H, \mathfrak{h})$ with the following properties.

(i) For every $h \in H$ we have $\sigma(h)=\pi \circ \phi(h)$.

(ii) For every $x \in \mathfrak{h}$ we have $\left.\rho^{\sigma}(x)\right|_{\mathscr{L}}=\rho^{\pi} \circ \varphi(x)$.

\section{The oscillator representation of $\left(\widehat{\mathrm{OSp}}_{\text {res }}(\mathscr{K}), \widehat{\mathfrak{o s p}}_{\text {res }}(\mathscr{K})\right)$}

In this section we show that the oscillator representation of the restricted orthosymplectic Banach-Lie supergroup is an analytic unitary representation in the sense of Definition 4.1. To simplify the presentation, we have omitted some of the tedious computations. They can be done using the method of [Neeb 2010b, Section 9].

Let $\mathscr{K}=\mathscr{K}_{\overline{0}} \oplus \mathscr{K}_{\overline{1}}$ be a $\mathbb{Z}_{2}$-graded complex Hilbert space. For simplicity we assume that both $\mathscr{K}_{\overline{0}}$ and $\mathscr{K}_{\overline{1}}$ are infinite-dimensional. The case where one or both of these spaces are finite-dimensional is similar.

We denote the inner product of $\mathscr{K}$ by $\langle\cdot, \cdot\rangle$. By restriction of scalars we can also consider $\mathscr{K}$ as a real Hilbert space.

Let $J_{+}: \mathscr{K} \rightarrow \mathscr{K}$ denote multiplication by $\sqrt{-1}$, and $J_{-}: \mathscr{K} \rightarrow \mathscr{K}$ be the map defined by

$$
J_{-} v=-(-1)^{p(v)} \sqrt{-1} v \text { for every homogeneous } v \in \mathscr{K} .
$$

In the following, both $J_{+}$and $J_{-}$will be considered as $\mathbb{R}$-linear maps.

If $A: \mathscr{K} \rightarrow \mathscr{K}$ is an $\mathbb{R}$-linear map, then $A J_{+}-J_{+} A$ is $\mathbb{C}$-conjugate linear. The space of Hilbert-Schmidt $\mathbb{R}$-linear maps on $\mathscr{K}$ (respectively, on $\mathscr{K}_{s}$, where $s \in\{\overline{0}, \overline{1}\}$ ) is denoted by $\mathrm{HS}(\mathscr{K})$ (respectively, by $\mathrm{HS}\left(\mathscr{K}_{S}\right)$ ). The group of bounded invertible $\mathbb{R}$-linear maps on $\mathscr{K}$ (respectively, on $\mathscr{K}_{s}$, where $s \in\{\overline{0}, \overline{1}\}$ ) is denoted by GL( $\left.\mathscr{K}\right)$ 
(respectively, by GL( $\left.\mathscr{H}_{s}\right)$ ). We set

$$
\mathrm{GL}_{\mathrm{res}}(\mathscr{K})=\left\{T \in \mathrm{GL}(\mathscr{K}): T J_{+}-J_{+} T \in \operatorname{HS}(\mathscr{K})\right\} .
$$

The groups $\mathrm{GL}_{\mathrm{res}}\left(\mathscr{K}_{s}\right)$, where $s \in\{\overline{0}, \overline{1}\}$, are defined similarly.

Let $\operatorname{End}_{\mathbb{R}}(\mathscr{K})=\operatorname{End}_{\mathbb{R}}(\mathscr{K})_{\overline{0}} \oplus \operatorname{End}_{\mathbb{R}}(\mathscr{K})_{\overline{1}}$ denote the superalgebra of bounded $\mathbb{R}$ linear maps on $\mathscr{K}$. Every $T \in \operatorname{End}_{\mathbb{R}}(\mathscr{K})$ can be written in a unique way as $T=$ $T_{\text {lin }}+T_{\text {conj }}$, where $T_{\text {lin }}$ is $\mathbb{C}$-linear and $T_{\text {conj }}$ is $\mathbb{C}$-conjugate linear. In fact we have

$$
T_{\text {lin }}=\frac{1}{2}\left(T-J_{+} T J_{+}\right) \quad \text { and } \quad T_{\text {conj }}=\frac{1}{2}\left(T+J_{+} T J_{+}\right) .
$$

The inner product of the complex Hilbert space $\mathscr{K}$ yields $\mathbb{R}$-bilinear forms $(\cdot, \cdot)_{\overline{0}}$ on $\mathscr{K}_{\overline{0}}$ and $(\cdot, \cdot)_{\overline{1}}$ on $\mathcal{K}_{\overline{1}}$ defined by

$$
\begin{array}{ll}
(v, w)_{\overline{0}}=\operatorname{Re}\langle v, w\rangle & \text { for every } v, w \in \mathscr{K}_{\overline{0}}, \\
(v, w)_{\overline{1}}=\operatorname{Im}\langle v, w\rangle & \text { for every } v, w \in \mathscr{K}_{\overline{1}} .
\end{array}
$$

As a result, we obtain an $\mathbb{R}$-bilinear form $(\cdot, \cdot)=(\cdot, \cdot)_{\overline{0}} \oplus(\cdot, \cdot)_{\overline{1}}$ on $\mathscr{K}=\mathscr{K}_{\overline{0}} \oplus \mathscr{K}_{\overline{1}}$.

The restricted orthogonal group $\mathrm{O}_{\mathrm{res}}\left(\mathscr{K}_{\overline{0}}\right)$ is defined by

$$
\mathrm{O}_{\text {res }}\left(\mathscr{K}_{\overline{0}}\right)=\left\{T \in \mathrm{GL}_{\text {res }}\left(\mathscr{K}_{\overline{0}}\right):(T v, T w)_{\overline{0}}=(v, w)_{\overline{0}} \quad \text { for every } v, w \in \mathscr{K}_{\overline{0}}\right\} .
$$

Observe that

$$
\mathfrak{o}_{\text {res }}\left(\mathscr{K}_{\overline{0}}\right)=\operatorname{Lie}\left(\mathrm{O}_{\text {res }}\left(\mathscr{K}_{\overline{0}}\right)\right)=\left\{T \in \mathfrak{g l}_{\text {res }}\left(\mathscr{K}_{\overline{0}}\right):(T v, w)_{\overline{0}}+(v, T w)_{\overline{0}}=0\right\},
$$

where

$$
\mathfrak{g l}_{\text {res }}\left(\mathscr{K}_{\overline{0}}\right)=\left\{T \in \operatorname{End}_{\mathbb{R}}\left(\mathscr{K}_{\overline{0}}\right): T_{\text {conj }} \in \operatorname{HS}\left(\mathscr{K}_{\overline{0}}\right)\right\} .
$$

The definitions of the restricted symplectic group $\mathrm{Sp}_{\mathrm{res}}\left(\mathscr{K}_{\overline{1}}\right)$ and its Lie algebra $\mathfrak{s p}_{\text {res }}\left(\mathscr{K}_{\overline{1}}\right)=\operatorname{Lie}\left(\operatorname{Sp}_{\text {res }}\left(\mathscr{K}_{\overline{1}}\right)\right)$ are analogous.

The Banach-Lie superalgebra $\mathfrak{o s p}_{\text {res }}(\mathscr{K})$ is the subspace of $\operatorname{End}_{\mathbb{R}}(\mathscr{K})$ spanned by elements $T \in \operatorname{End}_{\mathbb{B}}(\mathscr{K})_{\overline{0}} \cup \operatorname{End}_{\mathbb{R}}(\mathscr{K})_{\overline{1}}$ with the following properties.

(i) For every $v, w \in \mathscr{K}$, we have $(T v, w)+(-1)^{p(T) p(v)}(v, T w)=0$.

(ii) $T_{\text {conj }} \in \operatorname{HS}(\mathscr{K})$.

The norm $\|\cdot\|$ on $\mathfrak{o s p}_{\text {res }}(\mathscr{K})$ is given as follows. For every $T \in \mathfrak{o s p}_{\text {res }}(\mathscr{K})$, we set

$$
\|T\|^{\prime}=\left\|T_{\text {lin }}\right\|_{\mathrm{Op}}+\left\|T_{\text {conj }}\right\|_{\mathrm{HS}},
$$

where $\|\cdot\|_{\text {Op }}$ denotes the operator norm of a $\mathbb{C}$-linear operator on the (complex) Hilbert space $\mathscr{K}$ and $\|\cdot\|_{\mathrm{HS}}$ denotes the Hilbert-Schmidt norm of an $\mathbb{R}$-linear operator on the (real) Hilbert space $\mathscr{K}$. One can prove that $\|\cdot\|^{\prime}$ is continuous, and therefore by a suitable scaling one obtains a norm $\|\cdot\|$ which satisfies (3). 
The restricted orthosymplectic Banach-Lie supergroup associated to $\mathscr{K}$ is the Banach-Lie supergroup $\left(\operatorname{OSp}_{\text {res }}(\mathscr{K}), \mathfrak{o s p}_{\text {res }}(\mathscr{K})\right)$, where

$$
\operatorname{OSp}_{\text {res }}(\mathscr{K})=\mathrm{O}_{\text {res }}\left(\mathscr{K}_{\overline{0}}\right) \times \operatorname{Sp}_{\text {res }}\left(\mathscr{K}_{\overline{1}}\right) \text {. }
$$

It is known [Segal 1981] that to realize the spin representation of $\mathrm{O}_{\text {res }}\left(\mathscr{K}_{\overline{0}}\right)$ or the metaplectic representation of $\mathrm{Sp}_{\text {res }}\left(\mathscr{K}_{\overline{1}}\right)$ one needs to pass to certain central extensions $\widehat{\mathrm{O}}_{\text {res }}\left(\mathscr{K}_{\overline{0}}\right)$ and $\widehat{\mathrm{Sp}}_{\text {res }}\left(\mathscr{K}_{\overline{1}}\right)$ which are also Banach-Lie groups [Neeb 2010b, Section 9]. This leads to a Banach-Lie supergroup $\left(\widehat{\mathrm{OSp}}_{\text {res }}(\mathscr{K}), \widehat{\mathfrak{o s p}}_{\text {res }}(\mathscr{K})\right)$, where the Banach-Lie superalgebra $\widehat{\mathfrak{o s p}}_{\text {res }}(\mathscr{K})$ is the central extension of $\mathfrak{o s p}_{\text {res }}(\mathscr{K})$ corresponding to the cocycle

$$
\omega: \mathfrak{o s p}_{\text {res }}(\mathscr{K}) \times \mathfrak{o s p}_{\text {res }}(\mathscr{K}) \rightarrow \mathbb{R}
$$

which can be uniquely identified by the following properties.

(i) If $A, B \in \mathfrak{o s p}_{\text {res }}(\mathscr{K})$ have different parity then $\omega(A, B)=0$.

(ii) If $A, B \in \mathfrak{o s p}_{\text {res }}(\mathscr{K})_{\overline{0}}$ then $\omega(A, B)=-\frac{1}{2} \operatorname{tr}\left(J_{+} A_{\text {conj }} B_{\text {conj }}\right)$.

(iii) If $A, B \in \mathfrak{o s p}_{\text {res }}(\mathcal{K})_{\overline{1}}$ then $\omega(A, B)=-\frac{1}{2} \operatorname{tr}\left(J_{-} A_{\text {conj }} B_{\text {conj }}\right)$.

In other words, we have $\widehat{\mathfrak{o s p}}_{\text {res }}(\mathscr{K})=\mathfrak{o s p}_{\text {res }}(\mathscr{K}) \oplus \mathbb{R}$ as a vector space, with the superbracket

$$
\left[(T, z),\left(T^{\prime}, z^{\prime}\right)\right]=\left(\left[T, T^{\prime}\right], \omega\left(T, T^{\prime}\right)\right) .
$$

We now describe the Fock space realization of the metaplectic representation of $\widehat{\mathfrak{o s p}}_{\text {res }}(\mathscr{K})$. We choose an orthonormal basis $\left\{f_{1}, f_{2}, f_{3}, \ldots\right\}$ for the fermionic space $\mathscr{K}_{\overline{0}}$ and an orthonormal basis $\left\{b_{1}, b_{2}, b_{3}, \ldots\right\}$ for the bosonic space $\mathscr{K}_{\overline{1}}$. For every two integers $k, l \geq 0$, we define $\mathscr{F}^{k, l}$ to be the complex vector space spanned by monomials

$$
f_{1}^{r_{1}} f_{2}^{r_{2}} f_{3}^{r_{3}} \cdots b_{1}^{s_{1}} b_{2}^{s_{2}} b_{3}^{s_{3}} \cdots
$$

with the following properties.

(i) For every positive integer $m$, we have $r_{m} \in\{0,1\}$ and $s_{m} \in\{0,1,2,3, \ldots\}$.

(ii) For all but finitely many $m$, we have $r_{m}=s_{m}=0$.

(iii) $\sum_{m=1}^{\infty} r_{m}=k$ and $\sum_{m=1}^{\infty} s_{m}=l$.

We will refer to the monomials satisfying the above properties as reduced monomials. To simplify the notation, we will also use more general monomials of the form $v_{1} \cdots v_{m}$, where $v_{k} \in \mathscr{K}_{\overline{0}} \cup \mathscr{K}_{\overline{1}}$ for every $1 \leq k \leq n$. Observe that any such monomial can be expressed as a linear combination of reduced monomials using linearity and the relations

$$
f_{m} f_{n}=-f_{n} f_{m} \quad \text { and } \quad b_{m} b_{n}=b_{n} b_{m}
$$


for every two nonnegative integers $m, n$.

We set $\mathscr{F}=\bigoplus_{k, l \geq 0} \mathscr{F}^{k, l}$ and define an inner product $\langle\cdot, \cdot\rangle_{\mathscr{F}}$ on $\mathscr{F}$ as follows. If $v=f_{1}^{r_{1}} f_{2}^{r_{2}} \cdots b_{1}^{s_{1}} b_{2}^{s_{2}} \cdots \in \mathscr{F}^{k, l}$ and $w=f_{1}^{r_{1}^{\prime}} f_{2}^{r_{2}^{\prime}} \cdots b_{1}^{s_{1}^{\prime}} b_{2}^{s_{2}^{\prime}} \cdots \in \mathscr{F}^{k^{\prime}, l^{\prime}}$ then

$$
\langle v, w\rangle_{\mathscr{F}}= \begin{cases}1 & \text { if } r_{k}=r_{k}^{\prime} \text { and } s_{k}=s_{k}^{\prime} \text { for every } k \geq 1, \\ 0 & \text { otherwise. }\end{cases}
$$

Next we describe the action of $\widehat{\mathfrak{o s p}}_{\text {res }}(\mathscr{K})$ on $\mathscr{F}$. Let $(T, z) \in \widehat{\mathfrak{o s p}}_{\text {res }}(\mathscr{H})$, where $T$ is a homogeneous element expressed in the form $T=T_{\text {lin }}+T_{\text {conj }}$. For every $v \in \mathscr{F}$ we set

$$
\rho^{\mathscr{F}}((T, z)) v=\rho^{\mathscr{F}}\left(T_{\text {lin }}\right) v+\rho^{\mathscr{F}}\left(T_{\text {conj }}\right) v+\sqrt{-1} z \cdot v,
$$

where $\rho^{\mathscr{F}}\left(T_{\text {lin }}\right)$ and $\rho^{\mathscr{F}}\left(T_{\text {conj }}\right)$ are defined as follows. If $v=v_{1} \cdots v_{m}$ is a monomial then

$$
\rho^{\mathscr{F}}\left(T_{\text {lin }}\right) v=\sum_{r=1}^{m}(-1)^{p\left(T_{\text {lin }}\right)\left(p\left(v_{1}\right)+\cdots+p\left(v_{r-1}\right)\right)} v_{1} \cdots v_{r-1}\left(T_{\text {lin }} v_{r}\right) v_{r+1} \cdots v_{m} .
$$

We also define $\rho^{\mathscr{F}}\left(T_{\text {conj }}\right) v$ by

$$
\rho^{\mathscr{F}}\left(T_{\text {conj }}\right) v=a\left(T_{\text {conj }}\right) v-a\left(T_{\text {conj }}\right)^{\dagger} v,
$$

where $a\left(T_{\text {conj }}\right): \mathscr{F} \rightarrow \mathscr{F}$ and $a\left(T_{\text {conj }}\right)^{\dagger}: \mathscr{F} \rightarrow \mathscr{F}$ are linear maps defined as follows. If $v \in \mathscr{F}^{k, l}$ then

$$
a\left(T_{\text {conj }}\right) v=\lambda_{k, l}\left(\sqrt{-1} \sum_{r=1}^{\infty}\left(T_{\text {proj }} b_{r}\right) b_{r}+\sum_{r=1}^{\infty}\left(T_{\text {proj }} f_{r}\right) f_{r}\right) v
$$

where $\lambda_{k, l}=\frac{1}{2} \sqrt{(k+l+1)(k+l+2)}$. Moreover, $a\left(T_{\text {conj }}\right)^{\dagger}$ is the superadjoint of $a\left(T_{\text {conj }}\right)$ on $\mathscr{F}$, that is,

$$
a\left(T_{\text {conj }}\right)^{\dagger}= \begin{cases}a\left(T_{\text {conj }}\right)^{*} & \text { if } T \text { is even, } \\ -\sqrt{-1} a\left(T_{\text {conj }}\right)^{*} & \text { if } T \text { is odd, }\end{cases}
$$

where $a\left(T_{\text {conj }}\right)^{*}$ is the adjoint of $a\left(T_{\text {conj }}\right)$ on $\mathscr{F}$, which is defined by

$$
\left\langle a\left(T_{\text {conj }}\right)^{*} w, w^{\prime}\right\rangle_{\mathscr{F}}=\left\langle w, a\left(T_{\text {conj }}\right) w^{\prime}\right\rangle_{\mathscr{F}} \quad \text { for every } w, w^{\prime} \in \mathscr{F} .
$$

The restriction of this action to $\widehat{\mathfrak{o s p}}_{\text {res }}(\mathscr{K})_{\overline{0}}$ is the tensor product of the spin representation of $\widehat{\mathfrak{o}}_{\text {res }}\left(\mathscr{K}_{\overline{0}}\right)$ and the metaplectic representation of $\widehat{\mathfrak{s p}}_{\text {res }}\left(\mathscr{K}_{\overline{1}}\right)$. This representation of $\widehat{\mathfrak{o s p}}_{\text {res }}(\mathscr{K})_{\overline{0}}$ integrates to an analytic unitary representation $(\pi, \mathscr{H})$ of $\widehat{\mathrm{OSp}}_{\text {res }}(\mathscr{K})$ on the completion of $\mathscr{F}$. For every $(T, z) \in \widehat{\mathfrak{o s p}}_{\text {res }}(\mathscr{K})$, the space $\mathscr{F}$ consists of analytic vectors for the operator $\rho^{\mathscr{F}}((T, z))$ [Neeb 2010b, Section 9]. From Lemma 2.2 it follows that $\left(\pi, \mathscr{H}, \mathscr{F}, \rho^{\mathscr{F}}\right)$ is a prerepresentation of $\left(\widehat{\mathrm{OSp}}_{\text {res }}(\mathscr{K}), \widehat{\mathfrak{o s p}}_{\text {res }}(\mathscr{K})\right)$. Consequently, Theorem 4.10 implies the following result. 
Theorem 5.1. Let $\overline{\mathscr{F}}$ be the Hilbert space completion of the Fock space $\mathscr{F}$ defined above. Then there exists a unique analytic unitary representation $\left(\sigma, \rho^{\sigma}, \overline{\mathscr{F}}\right)$ of $\left(\widehat{\mathrm{OSp}}_{\text {res }}(\mathscr{K}), \widehat{\mathfrak{o s p}}_{\mathrm{res}}(\mathscr{H})\right)$ with the following properties.

(i) $\mathscr{F} \subseteq \overline{\mathscr{F}}^{\omega}$, that is, every $v \in \mathscr{F}$ is an analytic vector for $(\sigma, \overline{\mathscr{F}})$.

(ii) $\left.\rho^{\sigma}(x)\right|_{\mathscr{F}}=\rho^{\mathscr{F}}(x)$ for every $x \in \widehat{\mathfrak{o s p}}_{\text {res }}(\mathscr{K})$.

\section{Appendix A. A smooth nonanalytic unitary representation}

The goal of this appendix is to give two examples: a smooth unitary representation of a Banach-Lie group without nonzero analytic vectors, and an analytic unitary representation of a Banach-Lie group without nonzero bounded vectors.

We start with the first example. In this example the Hilbert space of the representation is $\mathscr{H}=L^{2}([0,1], \mathbb{C})$ and $G$ is the additive group of a Banach space $\mathfrak{g}$ of measurable functions $[0,1] \rightarrow \mathbb{R}$ with the property that

$$
L^{\infty}([0,1], \mathbb{R}) \subseteq \mathfrak{g} \subseteq \bigcap_{p \in \mathbb{N}} L^{p}([0,1], \mathbb{R}) .
$$

Using results from [Neeb 2010a], these two inclusions easily imply that the representation $(\pi, \mathscr{H})$ of $G$ given by $(\pi(f) \xi)(x)=e^{i f(x)} \xi(x)$ is smooth. Now the main point is to choose $\mathfrak{g}$ large enough so that the space $\mathscr{H}^{\omega}$ does not contain nonzero vectors.

Let $g(x)=e^{-\sqrt{x}}$ for $x \geq 0$. Then $\int_{0}^{\infty} x^{n} g(x) d x=2 \cdot \Gamma(2 n+2)<\infty$ for every $n \in \mathbb{N}$, while $\int_{0}^{\infty} e^{t x} g(x) d x=\infty$ for every $t>0$. Consider the map

$$
G:[0, \infty) \rightarrow[0, \infty), \quad G(x)=\frac{1}{2} \int_{0}^{x} g(t) d t .
$$

As $g$ is continuous, the function $G$ is $C^{1}$ with $G^{\prime}(x)>0$ for every $x \geq 0$. Next observe that

$$
\lim _{x \rightarrow \infty} G(x)=\frac{1}{2} \int_{0}^{\infty} e^{-\sqrt{t}} d t=\int_{0}^{\infty} e^{-x} x d x=\Gamma(2)=1 .
$$

Therefore $G:[0, \infty) \rightarrow[0,1)$ is a $C^{1}$-diffeomorphism. Set

$$
h(x)= \begin{cases}G^{-1}(1-x) & \text { if } 0<x \leq 1, \\ 0 & \text { if } x=0 .\end{cases}
$$

Then $h:[0,1] \rightarrow[0, \infty)$ is a Lebesgue measurable function with a singularity at 0 .

In the following we denote the Lebesgue measure of a measurable set $E \subseteq[0,1]$ by $\mu(E)$. We say that the metric density of $E$ at $x_{0}$ is 1 if

$$
\lim _{\varepsilon \rightarrow 0} \frac{\mu\left(E \cap\left[x_{0}-\varepsilon, x_{0}+\varepsilon\right]\right)}{2 \varepsilon}=1 .
$$


According to [Rudin 1987, §7.12], at almost every point of $E$ the metric density of $E$ is 1 . Clearly, 0 and 1 can never have this property for $E \subseteq[0,1]$. Note that at every point $x_{0}$ of metric density 1 , we also have

$$
\lim _{\varepsilon \rightarrow 0} \frac{\mu\left(E \cap\left[x_{0}, x_{0}+\varepsilon\right]\right)}{\varepsilon}=1 .
$$

Lemma A.1. If $\left\{a_{n}\right\}_{n=0}^{\infty}$ and $\left\{s_{n}\right\}_{n=0}^{\infty}$ are sequences of nonnegative real numbers such that $\left\{a_{n}\right\}_{n=0}^{\infty}$ is decreasing and $a_{n} \rightarrow 0$, then

$$
\sum_{n=0}^{\infty}\left(a_{n}-a_{n+1}\right)\left(s_{0}+\cdots+s_{n}\right)=\sum_{n=0}^{\infty} a_{n} s_{n} .
$$

Proof. For every two nonnegative integers $p, q$ set

$$
b_{p, q}= \begin{cases}\left(a_{p}-a_{p+1}\right) s_{q} & \text { if } p \geq q \\ 0 & \text { otherwise. }\end{cases}
$$

By [Rudin 1987, Corollary 1.27] we have $\sum_{p=0}^{\infty} \sum_{q=0}^{\infty} b_{p, q}=\sum_{q=0}^{\infty} \sum_{p=0}^{\infty} b_{p, q}$. The lemma follows easily from the latter equality.

Lemma A.2. Let $H:(0,1] \rightarrow \mathbb{R}$ be a continuous and decreasing map such that $\int_{0}^{1} H(x) d x=\infty$ and $E \subseteq[0,1]$ be a measurable set such that

$$
\lim _{\varepsilon \rightarrow 0} \frac{\mu(E \cap[0, \varepsilon])}{\varepsilon}=1 .
$$

Then $\int_{E} e^{t H(x)} d x=\infty$ for every $t>0$.

Proof. Our assumption implies that $\lim _{x \rightarrow 0} H(x)=\infty$ because otherwise $H$ would be bounded, hence integrable. Adding a constant to $H$ will not affect the statement of the lemma. Therefore we may assume that $H(1)=1$. We now put $\varepsilon_{n}:=H^{-1}\left(2^{n}\right)$ and note that $\varepsilon_{0}=1$ as well as $\varepsilon_{n} \rightarrow 0$. We now find that

$$
\infty=\int_{0}^{1} H(x) d x=\sum_{n=0}^{\infty} \int_{\varepsilon_{n+1}}^{\varepsilon_{n}} H(x) d x \leq \sum_{n=0}^{\infty} 2^{n+1}\left(\varepsilon_{n}-\varepsilon_{n+1}\right) .
$$

Lemma A.1 implies that

$$
\sum_{k=0}^{\infty} \varepsilon_{k} 2^{k}=\sum_{n=0}^{\infty}\left(2^{n+1}-1\right)\left(\varepsilon_{n}-\varepsilon_{n+1}\right)=\infty
$$

because $\sum_{n=0}^{\infty} \varepsilon_{n}-\varepsilon_{n+1}=\varepsilon_{0}$. 
If $E_{n}=E \cap\left[0, \varepsilon_{n}\right]$ for every $n \geq 0$, then using Lemma A.1 we have

$$
\begin{aligned}
\int_{E} H(x) d x & =\sum_{n=0}^{\infty} \int_{E_{n} \backslash E_{n+1}} H(x) d x \geq \sum_{n=0}^{\infty} 2^{n}\left(\mu\left(E_{n}\right)-\mu\left(E_{n+1}\right)\right) \\
& =\sum_{n=0}^{\infty}\left(1+1+2^{1}+\cdots+2^{n-1}\right)\left(\mu\left(E_{n}\right)-\mu\left(E_{n+1}\right)\right) \\
& =\mu\left(E_{0}\right)+\sum_{n=1}^{\infty} 2^{n-1} \mu\left(E_{n}\right) .
\end{aligned}
$$

Since $\lim _{n \rightarrow \infty} \mu\left(E_{n}\right) / \varepsilon_{n}=1$, from (34) it follows that $\int_{E} H(x) d x=\infty$.

Lemma A.3. The map $h$ given in (33) has the following properties:

(i) $h \in \bigcap_{p \in \mathbb{N}} L^{p}([0,1], \mathbb{R})$.

(ii) $\left.h\right|_{(0,1]}$ is strictly decreasing with $\lim _{x \rightarrow 0} h(x)=\infty$.

(iii) If $E \subseteq[0,1]$ is a measurable subset satisfying $\lim _{\varepsilon \rightarrow 0} \frac{\mu(E \cap[0, \varepsilon])}{\varepsilon}=1$, then $\int_{E} e^{t h(x)} d x=\infty$ for every $t>0$.

Proof. (i) For every $n \in \mathbb{N}$ we have

$$
\begin{aligned}
\int_{0}^{1} h(x)^{n} d x=\int_{0}^{1} h(1-x)^{n} d x & =\int_{0}^{\infty} h(1-G(y))^{n}\left|G^{\prime}(y)\right| d y \\
& =\frac{1}{2} \int_{0}^{\infty} y^{n} g(y) d y<\infty .
\end{aligned}
$$

(ii) Follows from the definition of $h$.

(iii) For every $t>0$ we have

$$
\begin{aligned}
\int_{0}^{1} e^{t h(x)} d x=\int_{0}^{1} e^{t h(1-x)} d x & =\int_{0}^{\infty} e^{t h(1-G(y))}\left|G^{\prime}(y)\right| d y \\
& =\frac{1}{2} \int_{0}^{\infty} e^{t y} g(y) d y=\infty .
\end{aligned}
$$

Lemma A.2 completes the argument.

Let $\|\cdot\|_{p}$ denote the usual norm of $L^{p}([0,1], \mathbb{R})$. Set $c_{n}=\|h\|_{n}$ for every $n \in \mathbb{N}$. Note that $c_{n}>0$ for every $n \in \mathbb{N}$. Since $h$ is unbounded, for every $c>0$ the set

$$
I_{c}=\{x \in[0,1]:|h(x)| \geq c\}
$$

has positive measure. This implies that $\|h\|_{n} \geq \sqrt[n]{\mu\left(I_{c}\right)} c$. Since the right hand side converges to $c$ when $n \rightarrow \infty$, it follows that $\lim _{n \rightarrow \infty}\|h\|_{n}=\infty$. 
We are now ready to define the Banach space $\mathfrak{g}$. For every measurable function $f:[0,1] \rightarrow \mathbb{R}$ we define a norm

$$
\|f\|=\sup \left\{\frac{\|f\|_{n}}{c_{n}}: n \in \mathbb{N}\right\},
$$

and set

$$
\mathfrak{g}=\left\{f \in \bigcap_{p \in \mathbb{N}} L^{p}([0,1], \mathbb{R}):\|f\|<\infty\right\} .
$$

It is fairly straightforward to check that $\mathfrak{g}$ is a Banach space and $L^{\infty}([0,1], \mathbb{R}) \subseteq \mathfrak{g}$. We set $G=\mathfrak{g}$, that is, the additive group of the Banach space $\mathfrak{g}$.

By construction, $h \in \mathfrak{g}$. Next we observe that we may also identify $\mathfrak{g}$ with a space of 1-periodic functions on $\mathbb{R}$. Then the norm defined on $\mathfrak{g}$ is translation invariant. Therefore $\mathfrak{g}$ also contains the functions $h_{x_{0}}(\cdot)=\left.\tilde{h}\left(\cdot-x_{0}\right)\right|_{[0,1]}$, where $\tilde{h}$ is the 1 -periodic extension of $h$ to $\mathbb{R}$. For $x_{0}<1$ and $0<\varepsilon<1-x_{0}$, it satisfies

$$
\int_{x_{0}}^{x_{0}+\varepsilon} e^{t h_{x_{0}}(x)} d x=\infty \quad \text { for } \quad t>0
$$

by Lemma A.3(iii).

Theorem A.4. Let $\mathscr{H}=L^{2}([0,1], \mathbb{C}), G=\mathfrak{g}$ be as above, and $(\pi, \mathscr{H})$ be the unitary representation of $G$ defined by $(\pi(f) \xi)(x)=e^{i f(x)} \xi(x)$. Then $(\pi, \mathscr{H})$ is smooth and $\mathscr{H}^{\omega}=\{0\}$.

Proof. According to [Neeb 2010a, Section 10] an element $\xi \in \mathscr{H}$ is a smooth vector if and only if

$$
\left\|f^{n} \xi\right\|_{2}<\infty \text { for every } n \in \mathbb{N} \text { and every } f \in \mathfrak{g} .
$$

Hence the inclusion $\mathfrak{g} \subseteq \bigcap_{p \in \mathbb{N}} L^{p}([0,1], \mathbb{R})$ implies that all bounded functions are smooth vectors. In particular, $(\pi, \mathscr{H})$ is a smooth representation.

By Lemma 3.2 and Theorem C.1 an element $\xi \in \mathscr{H}$ is analytic if and only if $\sum_{n=0}^{\infty}\left\|f^{n} \xi\right\|_{2} / n$ ! converges on some neighborhood of the origin in $\mathfrak{g}$. If $\xi$ is nonzero, then there exists an $\varepsilon>0$ for which the subset

$$
E=\{x \in[0,1]: \varepsilon<|\xi(x)|<1 / \varepsilon\}
$$

has positive measure. Let $\chi_{E}$ denote the characteristic function of the set $E$. Analyticity of $\xi$ leads to the estimates

$$
\int_{E} e^{|f(x)|} d x=\sum_{n=0}^{\infty} \frac{\int_{E}|f(x)|^{n} d x}{n !} \leq \sum_{n=0}^{\infty} \frac{\left\|f^{n} \chi_{E}\right\|_{2}}{n !} \leq \frac{1}{\varepsilon} \sum_{n=0}^{\infty} \frac{\left\|f^{n} \xi\right\|_{2}}{n !}<\infty
$$

for all elements $f$ in a neighborhood of the origin in $\mathfrak{g}$.

Let $x_{0} \in(0,1)$ be a point of metric density 1 of $E$ and recall that this implies that the sets $E_{\varepsilon}:=E \cap\left[x_{0}, x_{0}+\varepsilon\right]$ satisfy $\lim _{\varepsilon \rightarrow 0} \mu\left(E_{\varepsilon}\right) / \varepsilon=1$. Therefore Lemma A.2 
implies that the function $f(x)=\tilde{h}\left(x-x_{0}\right)$ satisfies $\int_{E} e^{t f(x)} d x=\infty$ for every $t>0$. This contradiction shows that there is no nonzero analytic vector in $\mathscr{H}$.

\section{Appendix B. An analytic representation without bounded vectors}

In this appendix we give an example of an analytic unitary representation of a Banach-Lie group without nonzero bounded vectors. The notation in this appendix is the same as in Appendix A.

Definition B.1. Let $G$ be a Banach-Lie group and $(\pi, \mathscr{H})$ be a unitary representation of $G$. We call $(\pi, \mathscr{H})$ bounded if $\pi: G \rightarrow \mathrm{U}(\mathscr{H})$ is continuous with respect to the operator norm on $\mathrm{U}(\mathscr{H})$. A vector $v \in \mathscr{H}$ is said to be bounded if the representation of $G$ on the closed invariant subspace $\mathscr{H}_{v}=\overline{\operatorname{Span}(\pi(G) v)}$ is bounded. The subspace of bounded vectors in $\mathscr{H}$ is denoted by $\mathscr{H}^{b}$. The representation $(\pi, \mathscr{H})$ is said to be locally bounded if $\mathscr{H}^{b}$ is dense in $\mathscr{H}$.

Since $G$ and $\mathrm{U}(\mathscr{H})$ are Banach-Lie groups, every bounded representation is in particular analytic as a map $G \rightarrow \mathrm{U}(\mathscr{H})$. In particular, $\mathscr{H}^{b} \subseteq \mathscr{H}^{\omega}$.

Observe that a representation is locally bounded if and only if it is a direct sum of bounded representations. In fact, if $\mathscr{H}^{b}$ is dense, then a standard application of Zorn's Lemma shows that $\mathscr{H}$ is an orthogonal direct sum of cyclic subspaces generated by bounded vectors.

For an element $x$ of a vector space $V$, we write $x^{*}(\alpha)=\alpha(x)$ for the corresponding linear functional on the dual space $V^{*}$.

Example B.2. (i) Let $G=(V,+)$ be the additive group of a finite-dimensional real vector space and $\mu$ be the Lebesgue measure on $V^{*}$. Then $\pi(x) f=e^{i x^{*}} f$ defines a continuous unitary representation on $\mathscr{H}=L^{2}\left(V^{*}, \mu\right)$. A vector $f \in \mathscr{H}$ is bounded if and only if $f$ vanishes almost everywhere outside some compact subset. Clearly this condition is stronger than $f \in \mathscr{H}^{\omega}$, which is equivalent to $e^{x^{*}} f$ being square integrable for every $x$ in a neighborhood of 0 in $V$.

(ii) When $G=(V,+)$ is the additive group of a finite-dimensional vector space, Bochner's theorem asserts that every continuous positive definite function $\phi: G \rightarrow$ $\mathbb{C}$ is the Fourier transform

$$
\phi=\hat{\mu}, \quad \hat{\mu}(x)=\int_{V^{\prime}} e^{i \alpha(x)} d \mu(\alpha)
$$

of a finite positive regular Borel measure $\mu$ on the dual space $V^{*}$. Then the representation of $\mathscr{H}=L^{2}\left(V^{*}, \mu\right)$ by $\pi(x) f=e^{i x^{*}} f$ is cyclic, generated by the constant function 1 , and $\langle\pi(x) 1,1\rangle=\hat{\mu}(x)=\phi(x)$. The description of the bounded and analytic vectors under (a) remains the same in this situation. 
(iii) If $G=(V,+)$ is the additive group of a Banach space and $\mu$ is a regular positive Borel measure on the topological dual space $V^{\prime}$ with respect to the weak* topology, then we also obtain a unitary representation of $G$ on $\mathscr{H}:=L^{2}\left(V^{\prime}, \mu\right)$ by $\pi(x) f=e^{i x^{*}} f$.

Every weak-*-compact subset of $V^{\prime}$ is weakly bounded, hence bounded by the Banach-Steinhaus theorem. Therefore the compact subsets of $V^{\prime}$ are precisely the weak-*-closed bounded subsets. All closed balls in $V^{\prime}$ have this property. If $\mu$ is supported by a bounded set, then one easily verifies that the representation $\pi$ is bounded. If this is not the case, then $f \in \mathscr{H}^{b}$ is equivalent to $f$ vanishing $\mu$-almost everywhere outside a sufficiently large ball. Since $\mu$ is regular, this implies that $\pi$ is locally bounded.

Theorem B.3. Let $G=(V,+)$ be the additive group of a Banach space. For a positive definite function $\phi$ on $G$ the corresponding $G N S$ representation $\left(\pi_{\phi}, \mathscr{H}_{\phi}\right)$ is locally bounded if and only if there exists a regular positive Borel measure $\mu$ on $V^{\prime}$ with $\phi=\hat{\mu}$.

Proof. If $\phi=\hat{\mu}$ for a regular positive Borel measure on $V^{\prime}$, then the GNS representation defined by $\phi$ is isomorphic to the cyclic subrepresentation of $L^{2}\left(V^{\prime}, \mu\right)$ generated by the constant function 1, hence locally bounded by Example B.2(iii).

Conversely, suppose that $\left(\pi_{\phi}, \mathscr{H}_{\phi}\right)$ is locally bounded, that is, it can be expressed as a direct sum

$$
\widehat{\bigoplus} \underset{j \in J}{ }\left(\pi_{j}, \mathscr{H}_{j}\right)
$$

of bounded representations. Writing $\phi=\sum_{j \in J} v_{j}$ with $v_{j} \in \mathscr{H}_{j}$, the orthogonality of the family $\left\{v_{j}: j \in J\right\}$ implies that only countably many of them are nonzero, and since $\phi$ is cyclic in $\mathscr{H}_{\phi}$, the index set $J$ is countable. Suppose that all the functions $\phi_{j}(g)=\left\langle\pi_{j}(g) v_{j}, v_{j}\right\rangle$ are Fourier transforms of positive regular Borel measures $\mu_{j}$ on $V^{\prime}$. Then $\phi=\sum_{j} \phi_{j}=\sum_{j} \hat{\mu}_{j}$ is the Fourier transform of the positive Borel measure $\mu=\sum_{j \in J} \mu_{j}$. Therefore without loss of generality we may assume that $\pi_{\phi}$ is a bounded representation. Then the spectral theorem for semibounded representations [Neeb 2009, Theorem 4.1] implies the existence of a regular Borel spectral measure $P$ on some weak-*-compact subset $X \subseteq V^{\prime}$ with $\pi_{\phi}(x)=\int_{X} e^{i \alpha(x)} d P(\alpha)$. For the cyclic vector $v \in \mathscr{H}_{\phi}$ satisfying $\phi(x)=\left\langle\pi_{\phi}(x) v, v\right\rangle$, this leads

$$
\phi(x)=\left\langle\pi_{\phi}(x) v, v\right\rangle=\int_{X} e^{i \alpha(x)} d P^{v}(\alpha)=\widehat{P^{v}}(x),
$$

where $P^{v}(E)=\langle P(E) v, v\rangle$ is the positive regular Borel measure associated to $v$ and $P$. 
The preceding discussion shows that the locally bounded cyclic representations are precisely those that can be realized in spaces $L^{2}\left(V^{\prime}, \mu\right)$ for regular Borel measures on $V^{\prime}$. For a representation $(\pi, \mathscr{H})$ with no nonzero bounded vector, for no nonzero $v \in \mathscr{H}$, the positive definite function $\pi^{v, v}(x)=\langle\pi(x) v, v\rangle$ is a Fourier transform of a regular Borel measure on $V^{\prime}$. In this sense they are very singular. In the light of this discussion, it is a natural question how big the difference between analytic and bounded representations really is.

From now on, for every measurable function $f$ we set

$$
\|f\|=\sup \left\{\frac{\|f\|_{n}}{\sqrt[n]{n !}: n \in \mathbb{N}\}} .\right.
$$

The following theorem shows that analytic representations of Banach-Lie groups need not contain nonzero bounded vectors.

Theorem B.4. The space

$$
\mathfrak{g}=\left\{f \in \bigcap_{p \in \mathbb{N}} L^{p}([0,1], \mathbb{R}):\|f\|<\infty\right\}
$$

is a Banach space. The unitary representation $\left(\pi, L^{2}([0,1], \mathbb{C})\right)$ of $G=(\mathfrak{g},+)$ given by $(\pi(f) \xi)(x)=e^{i f(x)} \xi(x)$ is analytic with $\mathscr{H}^{b}=\{0\}$.

Proof. To prove that $\mathfrak{g}$ is indeed a Banach space is straightforward and we leave it to the reader. As $\lim _{n \rightarrow \infty} \sqrt[n]{n !}=\infty$, we also have $L^{\infty}([0,1], \mathbb{R}) \subseteq \mathfrak{g}$, so that the constant function 1 is a cyclic vector. To show that $(\pi, \mathscr{H})$ is analytic, it therefore suffices to show that $1 \in \mathscr{H}^{\omega}$.

We claim that the series $\sum_{n=0}^{\infty} \frac{1}{n !}\left\|\mathrm{d} \pi(f)^{n} 1\right\|_{2}=\sum_{n=0}^{\infty} \frac{1}{n !}\left\|f^{n}\right\|_{2}$ converges uniformly for $\|f\|<\frac{1}{2}$, and this implies the analyticity of 1 . Below we need the estimate

$$
(2 n) !=(1 \cdots 3 \cdots(2 n-1))(2 \cdots 4 \cdots 2 n) \leq(2 \cdots 4 \cdots 2 n)^{2}=2^{2 n}(n !)^{2} .
$$

This leads to

$$
\sum_{n=0}^{\infty} \frac{1}{n !}\left\|f^{n}\right\|_{2}=\sum_{n=0}^{\infty} \frac{1}{n !}\|f\|_{2 n}^{n} \leq \sum_{n=0}^{\infty} \frac{\sqrt{(2 n) !}}{n !}\|f\|^{n} \leq \sum_{n=0}^{\infty} 2^{n}\|f\|^{n}=\frac{1}{1-2\|f\|} .
$$

Next we show that $\mathscr{H}^{b}=\{0\}$. Suppose that $\xi$ is a nonzero bounded vector, that is, that the representation of $G$ on the cyclic subspace $\mathscr{H}_{\xi}$ generated by $\xi$ is bounded. This implies in particular that $\mathscr{H}_{\xi}$ is invariant under the derived representation, that is, under multiplication with elements of $\mathfrak{g}$. As $\xi$ is nonzero, there exists an $\varepsilon>0$ for which the set $E=\{x \in[0,1]:|\xi(x)| \geq \varepsilon\}$ has positive measure. Since $\left(\left.\xi\right|_{E}\right)^{-1}$ is bounded, the characteristic function $\chi_{E}$ of $E$ is contained in $\mathscr{H}_{\xi}$, and further $L^{\infty}([0,1], \mathbb{R}) \cdot \chi_{E} \subseteq \mathscr{H}_{\xi}$ implies that $L^{2}(E) \subseteq \mathscr{H}_{\xi}$. 
The boundedness of the representation of $G$ on $L^{2}(E)$ implies in particular for each $f \in \mathfrak{g}$ that $\left\|e^{\left.t f\right|_{E}}-\chi_{E}\right\|_{\infty} \rightarrow 0$ for $t \rightarrow 0$, and hence that $\left.f\right|_{E}$ is essentially bounded. For $f(x)=\log (x)$ we have

$$
\|f\|_{n}^{n}=\int_{0}^{1}|\log (x)|^{n} d x=\int_{0}^{\infty} y^{n} e^{-y} d y=\Gamma(n+1)=n !
$$

which shows that $\log (\cdot) \in \mathfrak{g}$. Let $\widetilde{\log }$ denote the 1 -periodic extension of $\log$ to $\mathbb{R}$ and $\log _{x_{0}}(\cdot)=\left.\widetilde{\log }\left(\cdot-x_{0}\right)\right|_{[0,1]}$. Then the translation invariance of the norm defining $\mathfrak{g}$ implies that $\log _{x_{0}} \in \mathfrak{g}$.

As $E$ has positive measure, there exists a point $x_{0} \in(0,1) \cap E$ of metric density 1 , hence an $\varepsilon_{0}>0$ for which the set $E_{\varepsilon}=\left\{x \in E: x_{0} \leq x \leq x_{0}+\varepsilon\right\}$ has positive measure for every $\varepsilon \in\left(0, \varepsilon_{0}\right)$. This implies that $\log _{x_{0}}$ is not essentially bounded on $E$. From this contradiction we derive that $\mathscr{H}^{b}=\{0\}$.

\section{Appendix C. Analytic functions in the Banach context}

Let $\mathscr{A}$ and $\mathscr{B}$ be two Banach spaces over $\mathbb{K}$, where $\mathbb{K} \in\{\mathbb{R}, \mathbb{C}\}$. A homogeneous polynomial of degree $n$ from $\mathscr{A}$ to $\mathscr{B}$ is a map

$$
p: \mathscr{A} \rightarrow \mathscr{B}, \quad p(v)=F(v, \ldots, v),
$$

where $F: \mathscr{A} \times \cdots \times \mathscr{A} \rightarrow \mathscr{B}$ is a symmetric $\mathbb{K}$-multilinear map. The homogeneous polynomial $p$ is continuous if and only if $F$ is continuous.

If $U \subseteq \mathscr{A}$ is an open set, then a continuous function $f: U \rightarrow \mathscr{B}$ is called analytic in $U$ if and only if for every $u \in U$ there exist a neighborhood $V_{u}$ of the origin in $\mathscr{A}$ and continuous homogeneous polynomials $p_{n}: \mathscr{A} \rightarrow \mathscr{B}$ such that $\operatorname{deg}\left(p_{n}\right)=n$, $u+V_{u} \subseteq U$, and

$$
f(u+v)=\sum_{n=0}^{\infty} p_{n}(v) \quad \text { for every } v \in V_{u} .
$$

Remark. The convergence of the series (36) is pointwise. However, Theorem C.1 below implies that if the series (36) converges in $U$ pointwise, then for every $u \in U$ the series also converges normally at $u$ (i.e., absolutely uniformly in a neighborhood of $u$ ). In some references, e.g., [Bourbaki 1967, Number 3.2], analytic maps are defined on the basis of the latter form of convergence.

Recall that a subset $S$ of a vector space $\mathscr{V}$ over $\mathbb{K}$ is called absorbing if for every $v \in \mathscr{V}$ there exists a $t_{v}>0$ such that for all $c \in \mathbb{K}$, if $|c| \leq t_{v}$ then $c \cdot v \in S$.

Theorem C.1. Let $\mathscr{A}$ and $\mathscr{B}$ be Banach spaces over $\mathbb{K}$, where $\mathbb{K} \in\{\mathbb{R}, \mathbb{C}\}$. Let $S \subseteq \mathscr{A}$ be an absorbing set. For every integer $n \geq 0$, let $p_{n}: \mathscr{A} \rightarrow \mathscr{B}$ be a continuous 
homogeneous polynomial of degree $n$. Consider the formal series

$$
\phi(v)=\sum_{n=0}^{\infty} p_{n}(v) .
$$

(i) Suppose that there exists an absorbing set $S \subseteq \mathscr{A}$ such that the series $\phi(v)$ converges for every $v \in S$. Then there exists an open neighborhood $U$ of the origin in A such that

$$
\sum_{n=0}^{\infty} \sup \left\{\left\|p_{n}(v)\right\|: v \in U\right\}<\infty .
$$

(ii) Suppose that there exists an open neighborhood $V$ of the origin in $A$ such that the series $\phi(v)$ converges for every $v \in V$. Then the function

$$
\left.\phi\right|_{V}: V \rightarrow \mathscr{B}
$$

is analytic in $V$.

Proof. Statement (i) follows from [Bochnak and Siciak 1971b, Proposition 5.2 and Theorem 5.2]. Statement (ii) follows from [loc. cit., Theorem 5.2].

Remark. When $\mathscr{A}=\mathbb{C}^{n}$ and $\mathscr{B}=\mathbb{C}$, Theorem C.1 implies a result originally due to Hartogs. A proof of this special case is given in [Rudin 1980, Theorem 1.5.6].

Let $\mathscr{A}$ be a real Banach space and $\mathscr{B}$ be a complex Banach space (which can also be considered as a real Banach space). Every $\mathbb{R}$-multilinear map $F: \mathscr{A} \times \cdots \times \mathscr{A} \rightarrow$ $\mathscr{B}$ can be extended to a $\mathbb{C}$-multilinear map $F^{\mathbb{C}}: \mathscr{A}^{\mathbb{C}} \times \cdots \times \mathscr{A}^{\mathbb{C}} \rightarrow \mathscr{B}$ by extension of scalars. Therefore every continuous homogeneous polynomial $p: \mathscr{A} \rightarrow \mathscr{B}$ extends to a continuous homogeneous polynomial $p^{\mathbb{C}}: \mathscr{A}^{\mathbb{C}} \rightarrow \mathscr{B}$.

Theorem C.2. Let $\mathscr{A}$ be a real Banach space and $\Re$ be a complex Banach space. For every integer $n \geq 0$, let $p_{n}: \mathscr{A} \rightarrow \mathscr{B}$ be a continuous homogeneous polynomial of degree $n$. Suppose that the formal series

$$
\phi(v)=\sum_{n=0}^{\infty} p_{n}(v)
$$

converges for every $v \in U$, where $U$ is an open neighborhood of zero in $\mathscr{A}$. Then there exists an open neighborhood $U^{\mathbb{C}}$ of zero in $\mathscr{A}^{\mathbb{C}}$ such that $U \subseteq U^{\mathbb{C}}$, the series

$$
\phi^{\mathbb{C}}(v)=\sum_{n=0}^{\infty} p_{n}^{\mathbb{C}}(v)
$$

converges for every $v \in U^{\mathbb{C}}$, and the map $\left.\phi^{\mathbb{C}}\right|_{U^{\mathbb{C}}}: U^{\mathbb{C}} \rightarrow \mathscr{B}$ is analytic in $U^{\mathbb{C}}$. 
Proof. The statements of the theorem follow from [Bochnak and Siciak 1971b, Proposition 5.4] and Theorem C.1(ii) above.

An analogue of Hartogs' theorem is also valid in this framework.

Theorem C.3. Let $\mathscr{A}, \mathscr{B}$, and $\mathscr{C}$ be complex Banach spaces and $U \subseteq \mathscr{A} \times \mathscr{B}$ be an open set. If a function $f: U \rightarrow \mathscr{b}$ is separately analytic, then it is analytic.

Proof. This is [Bochnak and Siciak 1971b, Corollary 6.2].

Recall that in a complex topological vector space, a neighborhood $W$ of the origin is called balanced if and only if for every $z \in \mathbb{C}$ such that $|z| \leq 1$ and every $w \in W$ we have $z \cdot w \in W$.

Theorem C.4. Let $\mathscr{A}$ and $\mathscr{B}$ be complex Banach spaces, $U \subseteq \mathscr{A}$ be an open set, and $f: U \rightarrow \Re$ be analytic in $U$. Let $u \in U$ and $W$ be a balanced open neighborhood of zero in $A$ such that $u+W \subseteq U$. For every integer $n \geq 0$, set

$$
\delta_{u}^{(n)} f(v)=\left.\frac{d^{n}}{d \zeta^{n}} f(u+\zeta \cdot v)\right|_{\zeta=0} .
$$

Then the following statements hold.

(i) For every $n, \delta_{u}^{(n)} f$ is a continuous homogeneous polynomial of degree $n$.

(ii) $f(u+v)=\sum_{n=0}^{\infty} \frac{1}{n !} \delta_{u}^{(n)} f(v) \quad$ for every $v \in W$.

Proof. These statements are consequences of [Bochnak and Siciak 1971b, Proposition 5.5].

\section{References}

[Alldridge and Laubinger 2012] A. Alldridge and M. Laubinger, "Infinite-dimensional supermanifolds over arbitrary base fields", Forum Math. 24:3 (2012), 565-608. arXiv 0910.5430

[Alldridge et al. 2011] A. Alldridge, J. Hilgert, and M. Laubinger, "Harmonic analysis on Heisenberg-Clifford Lie supergroups", preprint, 2011. arXiv 1102.4475

[Bochnak and Siciak 1971a] J. Bochnak and J. Siciak, "Polynomials and multilinear mappings in topological vector spaces", Studia Math. 39 (1971), 59-76. MR 47 \#2364 Zbl 0214.37702

[Bochnak and Siciak 1971b] J. Bochnak and J. Siciak, "Analytic functions in topological vector spaces”, Studia Math. 39 (1971), 77-112. MR 47 \#2365 Zbl 0214.37703

[Boucher et al. 1986] W. Boucher, D. Friedan, and A. Kent, "Determinant formulae and unitarity for the $N=2$ superconformal algebras in two dimensions or exact results on string compactification", Phys. Lett. B 172:3-4 (1986), 316-322. MR 88e:17003 Zbl 1174.81319

[Bourbaki 1967] N. Bourbaki, Variétés différentielles et analytiques: fascicule de résultats (paragraphes 1 à 7), Actualités Scientifiques et Industrielles 1333, Hermann, Paris, 1967. MR 36 \#2161 Zbl 0171.22004

[Carmeli et al. 2006] C. Carmeli, G. Cassinelli, A. Toigo, and V. S. Varadarajan, "Unitary representations of super Lie groups and applications to the classification and multiplet structure of super particles”, Comm. Math. Phys. 263:1 (2006), 217-258. MR 2006m:22028 Zbl 1124.22007 
[Deligne and Morgan 1999] P. Deligne and J. W. Morgan, "Notes on supersymmetry (following Joseph Bernstein)", pp. 41-97 in Quantum fields and strings: a course for mathematicians (Princeton, NJ, 1996-1997), vol. 1, edited by P. Deligne et al., Amer. Math. Soc., Providence, RI, 1999. MR 2001g:58007 Zbl 1170.58302

[Dunford and Schwartz 1988] N. Dunford and J. T. Schwartz, Linear operators, II: Spectral theory, Wiley, New York, 1988. MR 90g:47001b Zbl 0635.47002

[Ferrara et al. 1981] S. Ferrara, C. A. Savoy, and B. Zumino, "General massive multiplets in extended supersymmetry", Phys. Lett. B 100:5 (1981), 393-398. MR 82f:81085

[Friedan et al. 1985] D. Friedan, Z. Qiu, and S. Shenker, "Superconformal invariance in two dimensions and the tricritical Ising model", Phys. Lett. B 151:1 (1985), 37-43. MR 86f:81087

[Goddard et al. 1986] P. Goddard, A. Kent, and D. Olive, "Unitary representations of the Virasoro and super-Virasoro algebras", Comm. Math. Phys. 103:1 (1986), 105-119. MR 87e:17018 Zbl 0588.17014

[Hille and Phillips 1974] E. Hille and R. S. Phillips, Functional analysis and semi-groups, Amer. Math. Soc. Colloquium Publications 31, Amer. Math. Soc., Providence, RI, 1974. MR 54 \#11077 Zbl 0392.46001

[Iohara 2010] K. Iohara, "Unitarizable highest weight modules of the $N=2$ super Virasoro algebras: untwisted sectors”, Lett. Math. Phys. 91:3 (2010), 289-305. MR 2011b:17054 Zbl 1228.17022

[Jakobsen and Kac 1989] H. P. Jakobsen and V. G. Kac, "A new class of unitarizable highest weight representations of infinite-dimensional Lie algebras, II", J. Funct. Anal. 82:1 (1989), 6990. MR 89m:17032 Zbl 0688.17007

[Jarvis and Zhang 1988] P. D. Jarvis and R. B. Zhang, "Unitary Sugawara constructions for affine superalgebras", Phys. Lett. B 215:4 (1988), 695-700. MR 90f:17039 Zbl 0689.17022

[Jørgensen and Moore 1984] P. E. T. Jørgensen and R. T. Moore, Operator commutation relations: commutation relations for operators, semigroups, and resolvents with applications to mathematical physics and representations of Lie groups, Reidel, Dordrecht, 1984. MR 86i:22006 Zbl 0535.47020

[Kac and Todorov 1985] V. G. Kac and I. T. Todorov, "Superconformal current algebras and their unitary representations”, Comm. Math. Phys. 102:2 (1985), 337-347. MR 87i:17021a Zbl 0599.17011

[Kirillov 1973] A. A. Kirillov, "Representations of the infinite-dimensional unitary group", Dokl. Akad. Nauk. SSSR 212 (1973), 288-290. In Russian; translated in Sov. Math. Dokl. 14 (1973), 13551358. MR 49 \#5239 Zbl 0288.22020

[Kostant 1977] B. Kostant, "Graded manifolds, graded Lie theory, and prequantization”, pp. 177306 in Differential geometrical methods in mathematical physics (Bonn, 1975), edited by K. Bleuler and A. Reetz, Lecture Notes in Math. 570, Springer, Berlin, 1977. MR 58 \#28326 Zbl 0358.53024

[Merigon 2011] S. Merigon, "Integrating representations of Banach-Lie algebras", J. Funct. Anal. 260:5 (2011), 1463-1475. MR 2012a:22036 Zbl 1226.22024

[Neeb 2009] K.-H. Neeb, "Semi-bounded unitary representations of infinite-dimensional Lie groups", pp. 209-222 in Infinite dimensional harmonic analysis, IV (Tokyo, 2007), edited by J. Hilgert et al., World Scientific, Hackensack, NJ, 2009. MR 2011g:22034 Zbl 1167.22013

[Neeb 2010a] K.-H. Neeb, "On differentiable vectors for representations of infinite dimensional Lie groups”, J. Funct. Anal. 259:11 (2010), 2814-2855. MR 2012b:22031 Zbl 1204.22016

[Neeb 2010b] K.-H. Neeb, "Semibounded representations and invariant cones in infinite dimensional Lie algebras", Confluentes Math. 2:1 (2010), 37-134. MR 2011g:22035 Zbl 1186.22023

[Neeb 2011] K.-H. Neeb, “On analytic vectors for unitary representations of infinite dimensional Lie groups”, Ann. Inst. Fourier 61:5 (2011), 1839-1874. Zbl 06032128 arXiv 1002.4792 
[Nelson 1959] E. Nelson, "Analytic vectors", Ann. of Math. (2) 70:3 (1959), 572-615. MR 21 \#5901 Zbl 0091.10704

[Ol'shanskii 1991] G. I. Ol'shanskiŭ, "Representations of infinite-dimensional classical groups, limits of enveloping algebras, and Yangians", pp. 1-66 in Topics in representation theory, edited by A. A. Kirillov, Adv. Soviet Math. 2, Amer. Math. Soc., Providence, RI, 1991. MR 92g:22039 Zbl 0739.22015

[Reed and Simon 1972] M. Reed and B. Simon, Methods of modern mathematical physics, I: Functional analysis, Academic Press, New York, 1972. MR 58 \#12429a Zbl 0242.46001

[Rudin 1980] W. Rudin, Function theory in the unit ball of $\mathbb{C}^{n}$, Grundlehren der Mathematischen Wissenschaften 241, Springer, New York, 1980. MR 82i:32002 Zbl 0495.32001

[Rudin 1987] W. Rudin, Real and complex analysis, 3rd ed., McGraw-Hill, New York, 1987. MR 88k:00002 Zbl 0925.00005

[Salam and Strathdee 1974] A. Salam and J. Strathdee, "Unitary representations of super-gauge symmetries”, Nuclear Phys. B 80:3 (1974), 499-505. MR 50 \#12029

[Salmasian 2010] H. Salmasian, "Unitary representations of nilpotent super Lie groups", Comm. Math. Phys. 297:1 (2010), 189-227. MR 2011f:22011 Zbl 1192.22003

[Sauvageot 1989] F. Sauvageot, "Représentations unitaires des super-algèbres de Ramond et de Neveu-Schwarz”, Comm. Math. Phys. 121:4 (1989), 639-657. MR 90h:17035 Zbl 0676.17017

[Segal 1981] G. Segal, "Unitary representations of some infinite dimensional groups", Comm. Math. Phys. 80:3 (1981), 301-342. MR 82k:22004 Zbl 0495.22017

[Siciak 1972] J. Siciak, "A polynomial lemma and analytic mappings in topological vector spaces", pp. 131-142 in Séminaire Pierre Lelong (Analyse) (Paris, 1970-1971), edited by P. Lelong, Lecture Notes in Math. 275, Springer, Berlin, 1972. MR 52 \#1307 Zbl 0237.58011

[Varadarajan 1999] V. S. Varadarajan, An introduction to harmonic analysis on semisimple Lie groups, Cambridge Studies in Advanced Mathematics 16, Cambridge University Press, Cambridge, 1999. MR 2000m:22011 Zbl 0924.22014

Received June 29, 2011. Revised August 25, 2011.

\section{STÉPHANE MERIGON}

DEPARTMENT OF MATHEMATICS

FAU ERLANGEN-NÜRNBERG

CAUERSTRASSE 11

91058 ERLANGEN

GERMANY

merigon@mi.uni-erlangen.de

KARL-HERMANN NEEB

DEPARTMENT OF MATHEMATICS

FAU ERLANGEN-NÜRNBERG

Cauerstrasse 11

91058 ERLANGEN

GERMANY

neeb@mi.uni-erlangen.de 
Department of Mathematics and Statistics

UNIVERSITY OF OTTAWA

585 King EDWARd AVENUE

OTTAWA K1N6N5

CANADA

hsalmasi@uottawa.ca 


\title{
PACIFIC JOURNAL OF MATHEMATICS
}

\author{
http://pacificmath.org \\ Founded in 1951 by \\ E. F. Beckenbach (1906-1982) and F. Wolf (1904-1989)
}

\section{EDITORS}

V. S. Varadarajan (Managing Editor)

Department of Mathematics

University of California

Los Angeles, CA 90095-1555

pacific@math.ucla.edu

Vyjayanthi Chari

Department of Mathematics

University of California

Riverside, CA 92521-0135

chari@math.ucr.edu

\section{Robert Finn}

Department of Mathematics Stanford University

Stanford, CA 94305-2125

finn@math.stanford.edu

Kefeng Liu

Department of Mathematics

University of California

Los Angeles, CA 90095-1555

liu@math.ucla.edu
Darren Long

Department of Mathematics

University of California

Santa Barbara, CA 93106-3080

long@math.ucsb.edu

Jiang-Hua Lu

Department of Mathematics

The University of Hong Kong

Pokfulam Rd., Hong Kong jhlu@maths.hku.hk

Alexander Merkurjev

Department of Mathematics

University of California

Los Angeles, CA 90095-1555

merkurev@math.ucla.edu
Sorin Popa

Department of Mathematics University of California

Los Angeles, CA 90095-1555 popa@math.ucla.edu

Jie Qing

Department of Mathematics

University of California

Santa Cruz, CA 95064

qing@cats.ucsc.edu

Jonathan Rogawski

Department of Mathematics

University of California

Los Angeles, CA 90095-1555

jonr@math.ucla.edu

\section{PRODUCTION}

pacific@math.berkeley.edu

\section{SUPPORTING INSTITUTIONS}

ACADEMIA SINICA, TAIPEI

CALIFORNIA INST. OF TECHNOLOGY INST. DE MATEMÁTICA PURA E APLICADA KEIO UNIVERSITY

MATH. SCIENCES RESEARCH INSTITUTE NEW MEXICO STATE UNIV.

OREGON STATE UNIV.

\author{
STANFORD UNIVERSITY \\ UNIV. OF BRITISH COLUMBIA \\ UNIV. OF CALIFORNIA, BERKELEY \\ UNIV. OF CALIFORNIA, DAVIS \\ UNIV. OF CALIFORNIA, LOS ANGELES \\ UNIV. OF CALIFORNIA, RIVERSIDE \\ UNIV. OF CALIFORNIA, SAN DIEGO \\ UNIV. OF CALIF., SANTA BARBARA
}

\author{
UNIV. OF CALIF., SANTA CRUZ \\ UNIV. OF MONTANA \\ UNIV. OF OREGON \\ UNIV. OF SOUTHERN CALIFORNIA \\ UNIV. OF UTAH \\ UNIV. OF WASHINGTON \\ WASHINGTON STATE UNIVERSITY
}

These supporting institutions contribute to the cost of publication of this Journal, but they are not owners or publishers and have no responsibility for its contents or policies.

See inside back cover or pacificmath.org for submission instructions.

The subscription price for 2012 is US \$420/year for the electronic version, and \$485/year for print and electronic.

Subscriptions, requests for back issues from the last three years and changes of subscribers address should be sent to Pacific Journal of Mathematics, P.O. Box 4163, Berkeley, CA 94704-0163, U.S.A. Prior back issues are obtainable from Periodicals Service Company, 11 Main Street, Germantown, NY 12526-5635. The Pacific Journal of Mathematics is indexed by Mathematical Reviews, Zentralblatt MATH, PASCAL CNRS Index, Referativnyi Zhurnal, Current Mathematical Publications and the Science Citation Index.

The Pacific Journal of Mathematics (ISSN 0030-8730) at the University of California, c/o Department of Mathematics, 969 Evans Hall, Berkeley, CA 94720-3840, is published monthly except July and August. Periodical rate postage paid at Berkeley, CA 94704, and additional mailing offices. POSTMASTER: send address changes to Pacific Journal of Mathematics, P.O. Box 4163, Berkeley, CA 94704-0163.

PJM peer review and production are managed by EditFLOW ${ }^{\mathrm{TM}}$ from Mathematical Sciences Publishers.

PUBLISHED BY PACIFIC JOURNAL OF MATHEMATICS

at the University of California, Berkeley 94720-3840

A NON-PROFIT CORPORATION

Typeset in LATEX

Copyright $(02012$ by Pacific Journal of Mathematics 


\section{PACIFIC JOURNAL OF MATHEMATICS}

Volume $257 \quad$ No. $2 \quad$ June 2012

Extending triangulations of the 2-sphere to the 3-disk preserving a

4-coloring

RUi PEDRO CARPENTIER

Orthogonal quantum group invariants of links

LIN CHEN and QINGTAO CHEN

Some properties of squeezing functions on bounded domains

FUSHENG DENG, QIAN GUAN and LIYOU ZHANG

Representations of little $q$-Schur algebras

JiE DU, QIANG FU and JiAN-PAN WANG

Renormalized weighted volume and conformal fractional Laplacians

MARÍA DEL MAR GONZÁLEZ

The $L_{4}$ norm of Littlewood polynomials derived from the Jacobi symbol 395

JONATHAN JEDWAB and KAI-UWE SCHMIDT

On a conjecture of Kaneko and Ohno

ZHONG-HUA LI

Categories of unitary representations of Banach-Lie supergroups and restriction functors

StéPhane Merigon, Karl-Hermann NeEb and Hadi

SALMASIAN

Odd Hamiltonian superalgebras and special odd Hamiltonian superalgebras of formal vector fields

Li Ren, Qiang Mu and YongZheng ZHANG

Interior derivative estimates for the Kähler-Ricci flow

Morgan SHERMAN and BEN WEINKOVE

Two-dimensional disjoint minimal graphs

LINFENG ZHOU 